\title{
THE INTEGRAL REPRESENTATION OF WEAKLY ALMOST-PERIODIC TRANSFORMATIONS IN REFLEXIVE VECTOR SPACES
}

BY

\author{
EDGAR R. LORCH
}

\section{INTRODUCTION}

The first analysis of the structure of transformations of a type somewhat similar to that treated below was given by $\mathrm{D}$. Hilbert in his memoir on bounded quadratic forms in infinitely many variables (1906). In the language freely used at present, Hilbert established that if $H$ is a bounded symmetric transformation in the (Hilbert) space of vectors $x=\left(x_{1}, x_{2}, \cdots\right)$ with $\sum\left|x_{\alpha}\right|^{2}<\infty$, then $H$ may be represented in the form

$$
H=\int \lambda d E(\lambda)
$$

The $E(\lambda)$ are orthogonal projections, that is to say, transformations which are the identity transformation on a closed linear manifold in the space and which are the zero transformation on the orthogonally complementary manifold. As $\lambda$ varies increasingly from some bound $-M$ to $M, E(\lambda)$ sweeps monotonously from the zero to the identity transformation. The values $\lambda,-M \leqq \lambda$ $\leqq M$, are classed as being regular or belonging to the "spectrum" of $H$ according to the behaviour of $E(\lambda)$. The complete analysis of the spectrum of $H$ leading to the integral representation (1) is sometimes described as establishing the spectral resolution of $H$. The formula (1) is the Hilbert space correspondent of the fundamental theorem of matrix theory to the effect that every symmetric $n \times n$ matrix may be reduced to diagonal form by a proper choice of axes.

Since the publication of Hilbert's result, the theory initiated by him has been elaborated in many directions. But the class of transformations in infinite dimensional spaces whose structure is thoroughly known has not grown. There is at least one important exception: the restriction of boundedness upon $H$ has been deleted. It is known as a result of the work of Carleman, von Neumann, F. Riesz, Stone, and Wintner $\left({ }^{1}\right)$, that the equation (1) sub-

Presented to the Society in two parts, the first under the title Spectral analysis of weakly almost periodic transformations in reflexive vector spaces on December 29,1939; the second, under the present title on October 26,1940; received by the editors May 8, 1940. Abstracted in the Bulletin of the Society, 45-11-401 and 46-7-361.

(1) Carleman, Sur les Équations Intégrales Singulières à Noyau Réel et Symétrique, Uppsala, 1923; J. von Neumann, Mathematische Annalen, vol. 102 (1929), pp. 49-131; F. Riesz, Acta Litterarum ac Scientiarum (Szeged), vol. 5 (1930), pp. 19-54; Stone, Linear Transforma- 
sists in the non-bounded case. Here the range of integration necessarily is not bounded on the $\lambda$ axis. Suggestive of a new point of departure is a result established by von Neumann $\left({ }^{2}\right)$ that a rotation or unitary transformation $V$ in Hilbert space has a "diagonal" representation of the form

$$
V=\int e^{i \lambda} d E(\lambda)
$$

Since simple correspondences may be established between unitary and symmetric (or better, self-adjoint) transformations, it is correct to state that the only transformations analyzed to the present (leaving aside certain considerably simpler types such as the completely continuous transformations) are the rotations in Hilbert space and the functions which they beget, e.g., $H=-i(V-I)(V+I)^{-1}, e^{H}$, etc.

A study of this theory reveals that its development is wedded to and completely dominated by the concept of orthogonality. It is the nature of this concept which renders impossible the mere extension of the notion of symmetric transformation to non-Hilbert spaces. Properly speaking, orthogonality within a space $\mathfrak{B}$ is meaningless; it is biorthogonality between $\mathfrak{B}$ and its adjoint space $(\mathfrak{B})$ which is significant. As Hilbert space is distinguished by the equation $\mathfrak{B}=(\mathfrak{B})$, biorthogonality is operative within the space itself and is then called orthogonality. Lacking a theory of orthogonality, entirely new methods have to be developed if one is to progress in the study, for example, of the structure of rotations in various non-Hilbert spaces. In the subsequent pages, the spectral resolution of a type of transformation in certain rather general spaces is obtained. All rotations fall under this type. But even in Hilbert space, new classes are analyzed for the first time.

The nature of the space for which the results are valid will first be described. For an arbitrary normed linear vector space $\mathfrak{B}$, the inclusion $((\mathfrak{B})) \supset \mathfrak{B}$ is easy to establish. A space distinguished by the relation

$$
\mathfrak{B}=((\mathfrak{B}))
$$

is called reflexive by the author. It is for reflexive spaces that a spectral theory is developed.

The precise nature of the transformation $V$ for which formula (2) may be established will be described briefly. Starting with a $V$ such that $V^{-1}$ exists (and is bounded), the set $\left\{V^{n} f\right\}, n=0, \pm 1, \pm 2, \cdots, f$ fixed in $\mathfrak{B}$, is examined. Borrowing ideas from the theory of almost-periodic functions, one defines $V$ to be weakly almost-periodic if the set $\left\{V^{n} f\right\}$ is weakly conditionally

tions in Hilbert Space and Their Applications to Analysis, American Mathematical Society Colloquium Publications, vol. 15, New York, 1932, chap. 5; Wintner, Spektraltheorie der unendlichen Matrizen, Leipzig, 1929, chap. 6.

() J. von Neumann, op. cit., p. 119. 
compact for all $f \boldsymbol{\varepsilon} \mathfrak{B}$. That is, if relative to the group $V^{n}$, all $f \boldsymbol{\varepsilon} \mathfrak{B}$ are almostperiodic, then $V$ is called almost-periodic-all this in the weak sense. The almost-periodicity of $V$ is characterized by the theorem: $V$ is weakly almost-periodic if and only if it is uniformly bounded, $\left\|V^{n} f\right\| \leqq K\|f\|, n=0$, $\pm 1, \pm 2, \cdots, f$ arbitrary. It is these transformations which are analyzed.

The results of the analysis yield a structure $\left\{\mathfrak{F}_{\lambda}, \mathfrak{F}_{\lambda}\right\},-\infty<\lambda<\infty$, strongly resembling a resolution of the identity and replacing it in the present instance. The $\mathfrak{F}_{\lambda}$ and $\mathfrak{F}_{\lambda}$ are closed linear manifolds having only the origin in common and together spanning $\mathfrak{B}$. Further, $\mathfrak{E}_{\lambda}\left(\mathfrak{F}_{\lambda}\right)$ is a monotone function of $\lambda$ increasing (decreasing) from $0(\mathfrak{B})$ to $\mathfrak{B}(0)$. The manifold pairs reduce $V$. Whether $\mathfrak{E}_{\lambda}$ and $\mathfrak{F}_{\lambda}$ are disjoint (see below) is yet to be determined. The reconstruction of $V$, in a sense justifying the use of (2), from the manifolds which it determines is carried out in Theorem 8 . The spectral character of $V$ is completely determined by the structure of $\left\{\mathfrak{F}_{\lambda}, \mathfrak{F}_{\lambda}\right\}$-Theorem 9. As an instance, $\exp (i \lambda),-\pi<\lambda<\pi$, is in the resolvent set of $V$ if and only if $\lambda$ is a point of constancy of $\left\{\mathfrak{F}_{\lambda}, \mathfrak{F}_{\lambda}\right\}$.

In the final chapter, the transformation $H=-i(V-I)(V+I)^{-1}$ is subjected to a thorough analysis. Formula (1) is established for it (Theorem 10), and it is shown that its spectrum may be read accurately in $\left\{\mathfrak{F}_{\lambda}, \mathfrak{F}_{\lambda}\right\}$-Theorem 11.

The present paper is based at least in part upon two previous papers of the author. The reader is referred to these at various points in the development. One of these papers, on the so-called mean-ergodic theorem in reflexive vector spaces, embodies the earliest results obtained in the present investigation, and quite naturally was to have been incorporated in the present paper (Theorems 3 and 4). However, the interest which similar theorems had aroused more recently made it desirable to publish the result separately (see footnote 7 ). In addition to these sources, no acquaintance with linear transformation theory is necessary to an understanding of the contents, other than may be found in the elementary chapters of Banach's treatise on linear operations.

\section{WEAKLY ALMOST-PERIODIC TRANSFORMATIONS}

The underlying space $\mathfrak{B}$ is assumed to be reflexive throughout (exceptions: Theorems 2 and $4^{\prime}$ ). That is to say, $\mathfrak{B}$ is a linear normed complex vector space satisfying the identity $\mathfrak{B}=((\mathfrak{B}))$. Here $(\mathfrak{B})$ denotes the space of all bounded linear functionals defined on $\mathfrak{B}$; $(\mathfrak{B})$ is called the space adjoint to $\mathfrak{B}$. Thus $((\mathfrak{B}))$ is the space adjoint to $(\mathfrak{B})$. Elements in $\mathfrak{B}$ will be denoted by $f, g, h, f_{n}, \eta, \zeta$, etc.; real numbers by $\lambda, \mu, \epsilon$, etc.; complex numbers by $\alpha, \beta$, etc. Transformations will be denoted by $T, V, A, B, P, I$ (the identity), and 0 (the zero), etc. They are linear or distributive, that is, $T(\alpha f+\beta g)=\alpha T f+\beta T g$. The bound of a transformation $T$ (if it exists) is denoted by $|T|$, also by $K, M$, etc. If $T$ is bounded and possesses a bounded inverse $T^{-1}\left(T T^{-1}\right.$ $\left.=T^{-1} T=I\right), T$ is said to be bicontinuous. 
If $T$ is bicontinuous, it generates a group of transformations, viz., $T^{n}$, $n=0, \pm 1, \pm 2, \cdots\left(T^{0}=I\right)$, with $T^{n} \cdot T^{m}=T^{n+m}$. A notion of the theory of almost-periodic functions suggests an analogue for the present study. A function $f(x)$ defined over a group is called almost-periodic if the set of functions $\{f(c x)\}$ with $c$ a parameter ranging over the group is conditionally compact. Consider now the set $\left\{T^{n} f\right\}, n=0, \pm 1, \pm 2, \cdots, f \varepsilon \mathfrak{B}$ fixed; whether the set is conditionally compact or not, that is, whether a converging subsequence can be drawn from any sequence in $\left\{T^{n} f\right\}$ depends on the type of convergence in question. Of the two types which instantly present themselves, strong and weak convergence, the latter seems much more fitting here. The significance of the definition which follows is now clear.

Definition. Let $T$ be a bicontinuous transformation in a reflexive space $\mathfrak{B}$. If the set $\left\{T^{n} f\right\}, n=0, \pm 1, \pm 2, \cdots, f$ fixed, is weakly conditionally compact for every $f \in \mathfrak{B}, T$ is said to be weakly almost-periodic.

A space is called weakly compact if every bounded set in the space is weakly conditionally compact. A space is called weakly complete if every weakly converging sequence converges weakly to an element of the space. It is known that reflexive spaces are both weakly compact and weakly complete $\left(^{3}\right)$. Since any weakly converging sequence is necessarily bounded $\left({ }^{4}\right)$ it follows that in order that $T$ be weakly almost-periodic (for short, w.a.p.), it is necessary and sufficient that for each $f$ the set $\left\{T^{n} f\right\}$ be bounded. Thus for every $f \varepsilon \mathfrak{B}$, there must exist a constant $K_{f}$ such that $\left\|T^{n} f\right\| \leqq K_{f}\|f\|, n=0, \pm 1$, $\pm 2, \cdots$.

A bicontinuous transformation $T$ is said to be uniformly bounded if the bound of $T^{n}$ satisfies the inequality $\left|T^{n}\right| \leqq K, n=0, \pm 1, \pm 2, \cdots$, for some constant $K$. The first theorem links the concepts of uniform boundedness and weak almost-periodicity.

THEOREM 1. In a reflexive space, the concepts of uniform boundedness and weak almost-periodicity are equivalent.

Let $T$ be uniformly bounded, $\left|T^{n}\right| \leqq K, n=0, \pm 1, \pm 2, \cdots$. Then for an arbitrary $f \varepsilon \mathfrak{B},\left\|T^{n} f\right\| \leqq K\|f\|$. Thus by the argument just given above, $T$ is w.a.p.

Now let $T$ be w.a.p. Then for $f \boldsymbol{\varepsilon} \mathfrak{B}$, there exists a constant $K_{f}$ such that $\left\|T^{n} f\right\| \leqq K_{f}\|f\|$. The existence of a single constant $K$ valid for all $f \varepsilon \mathfrak{B}$ will be established.

Let there be constructed a normed linear complex vector space $\mathbb{S}$ in the following fashion: The elements of $\mathbb{E}$ are the objects

$$
[f]=\left\{\cdots, T^{-2} f, T^{-1} f, f, T f, T^{2} f, \cdots\right\},
$$

( $\left.{ }^{3}\right)$ Pettis, $A$ note on regular spaces, Bulletin of the American Mathematical Society, vol. 44 (1938), pp. 420-428.

(4) Banach, Théorie des Opérations Linéaires, Warsaw, 1932, p. 133. 
$f \varepsilon \mathfrak{B}$. Multiplication by a complex scalar $\alpha$ is defined by $\alpha[f]=[\alpha f]$. Addition of vectors is defined by $[f]+[g]=[f+g]$. The norm of the vector $[f]$ is defined by

$$
\|[f]\|=\text { l.u.b. }\left\|T^{n} f\right\|, \quad n=0, \pm 1, \pm 2, \cdots \text {. }
$$

Clearly $\|[f]\| \leqq K_{f}\|f\|$.

The space $\mathbb{E}$ is complete. For let $\left\{[f]_{r}\right\}=\left\{\left[f_{r}\right]\right\}$ be a sequence converging in the above norm,

$$
\lim _{r, s \rightarrow \infty}\left\|\left[f_{r}\right]-\left[f_{s}\right]\right\|=\lim _{r, s \rightarrow \infty}\left\|\left[f_{r}-f_{s}\right]\right\|=0 .
$$

Thus $\lim _{r, s \rightarrow \infty}\left\|T^{n} f_{r}-T^{n} f_{s}\right\|=0$ uniformly in $n$. For $n=0$, it is seen that the sequence $\left\{f_{r}\right\}$ converges to some element $f \boldsymbol{\varepsilon} \mathfrak{B}$. Also the convergence of $\left\{T^{n} f_{r}\right\}$ to $T^{n} f$ is uniform in $n$. It is now clear that $\left\{\left[f_{r}\right]\right\}$ converges to $[f]$. For

$$
\left\|\left[f-f_{r}\right]\right\|=\underset{n}{\operatorname{liu.b}}\left\|T^{n}\left(f-f_{r}\right)\right\| \rightarrow 0
$$

with $r \rightarrow \infty$. Thus $\sqrt{5}$ is complete. Since

$$
\begin{aligned}
& \underset{n}{\text { l.u.b. }}\left\|T^{n}(f+g)\right\| \leqq \underset{n}{\operatorname{l.u.b} .}\left\|T^{n} f\right\|+\underset{n}{\text { l.u.b. }}\left\|T^{n} g\right\| \text {, } \\
& \|[f+g]\| \leqq\|[f]\|+\|[g]\| .
\end{aligned}
$$

Also $\|[\alpha f]\|=|\alpha| \cdot\|[f]\|$. Hence $\sqrt{5}$ is a normed linear complex vector space.

Now let $U$ be the mapping of the space $\mathfrak{B}$ upon the space $\mathbb{E}$ which carries $f \varepsilon \mathfrak{B}$ into $[f] \varepsilon \mathfrak{\varepsilon}$,

$$
\boldsymbol{U} f=[f]
$$

$\boldsymbol{U}$ is distributive. Furthermore, $\boldsymbol{U}$ is closed, that is, if $f_{r} \rightarrow f$ and $\left[f_{r}\right] \rightarrow[g]$, then $U f=[g]$. For since the sequence $\left\{\left[f_{r}\right]\right\}$ converges (which in itself implies that the sequence $\left\{f_{r}\right\}$ converges) and since $f_{r} \rightarrow f$, then $g=f$. This implies the closure of $U$.

It is known that a closed distributive transformation whose domain is the entire space $\mathfrak{B}$ is bounded $\left(^{5}\right)$. Thus $|\boldsymbol{U}| \leqq K$ and

$$
\left\|T^{n} f\right\| \leqq\|[f]\|=\|U f\| \leqq K\|f\| .
$$

This proves the theorem.

It is to be noted that all rotations, that is to say, all bicontinuous transformations $V$ which are isometric, $\|V f\|=\|f\|$, are w.a.p. So are also all periodic transformations. The structure of periodic linear transformations is particularly simple and is given in the next theorem. The results presage those attending the discussion of a general w.a.p. transformation.

(5) Banach, p. 41 . 
THEOREM 2. Let $T$ be a bounded linear periodic transformation of period $n$, $T^{n}=I$. Let $\left|T^{r}\right| \leqq K, r=1,2, \cdots, n$. Then there exist projections $P_{r}$, $r=1,2, \cdots, n$, with the properties:

(a) $\left|P_{r}\right| \leqq K ; P_{r} P_{s}=P_{s} P_{r}=0, r \neq s$.

(b) $P_{1}+P_{2}+\cdots+P_{n}=I$.

(c) $T P_{r} f=\alpha^{r} P_{r} f$ where $\alpha=e^{2 \pi i / n}$.

Note that $f=f_{1}+f_{2}+\cdots+f_{n}$ provided that

$$
f_{r}=\frac{1}{n}\left(f+\alpha^{-r} T f+\alpha^{-2 r} T^{2} f+\cdots+\alpha^{-(n-1) r} T^{n-1} f\right),
$$

$r=1,2, \cdots, n$. Clearly

$$
T f_{r}=\alpha^{r} f_{r}, \quad r=1,2, \cdots, n .
$$

The transformations $f \rightarrow f_{r}, P_{r} f=f_{r}$, are distributive.

$$
\left\|f_{r}\right\| \leqq \frac{1}{n}\left(\|f\|+\left\|\alpha^{-r} T f\right\|+\cdots+\left\|\alpha^{-(n-1) r} T^{n-1} f\right\|\right) \leqq K\|f\|,
$$

$\left|P_{r}\right| \leqq K$. That $P_{r}^{2} f=P_{r} f_{r}=f_{r}=P_{r} f$ may be ascertained directly from the definition of $f_{r}$. Thus the $P_{r}$ are projections. Similarly it may be seen that $P_{r} P_{s}=P_{s} P_{r}=0$ if $r \neq s$. Finally, that $P_{1}+P_{2}+\cdots+P_{n}=I$ is the opening statement of this proof.

\section{THE REDUCIBILITY OF WEAKLY ALMOST-PERIODIC TRANSFORMATIONS}

In the subsequent sections, a w.a.p. transformation will frequently be denoted by $V$. The spectral analysis of $V$ is based upon the following considerations. Let us assume that a representation (2) is possible for $V$. Let us grant that the "resolution of the identity" $E(\lambda)$ has a sufficiently smooth structure so that an operational calculus can be based upon it $\left(^{6}\right)$. Then in this calculus, $V$ corresponds to the function $\exp (i \lambda)$. This suggests that the structure of $V$ may be analyzed by means of Fourier series. Of course not any Fourier series is acceptable, but since $\left|V^{n}\right| \leqq K$, any series for which the series of coefficients is absolutely convergent is meaningful and may be useful in the present situation. A series of the type which "splits" $V$ will now be introduced.

Let $f(x)$ be a function of period $2 \pi$ defined by

$$
\begin{array}{rlrl}
f(x) & =0, & -\pi \leqq x \leqq 0 ; \\
f(x) & =\sin x, & 0 & <x<\pi .
\end{array}
$$

Let $\boldsymbol{g}(x)$ be defined by $\boldsymbol{g}(x)=\sin x-f(x)$. The Fourier expansion of $f(x)$ is

(8) Lorch, On a calculus of operators in reflexive vector spaces, these Transactions, vol. 45 (1939), pp. 217-234. See in particular Definition 4. 


$$
\begin{aligned}
f(x)= & \frac{1}{2} \sin x+\frac{2}{\pi}\left(\frac{1}{2}-\frac{\cos 2 x}{1 \cdot 3}-\frac{\cos 4 x}{3 \cdot 5}-\frac{\cos 6 x}{5 \cdot 7}-\cdots\right) \\
= & \frac{1}{4 i}\left(e^{i x}-e^{-i x}\right) \\
& +\frac{1}{\pi}\left(1-\frac{1}{1 \cdot 3}\left(e^{2 i x}+e^{-2 i x}\right)-\frac{1}{3 \cdot 5}\left(e^{4 i x}+e^{-4 i x}\right)-\cdots\right) .
\end{aligned}
$$

The function $\boldsymbol{g}(x)$ has a very similar development. For both functions the series of absolute values are uniformly convergent. Hence the expansion (5) converges to $\boldsymbol{f}(\boldsymbol{x})$; likewise for $\boldsymbol{g}(x)$. The two series may be multiplied and terms rearranged and grouped in any convenient fashion; their product is

$$
0=\boldsymbol{f}(x) \cdot g(x)=\sum_{-\infty}^{\infty} a_{r} e^{i r x}
$$

By the Heine-Cantor theorem, $a_{r}=0,-\infty<r<\infty$. As a matter of fact a very elementary reasoning involving term-by-term integration gives $a_{r}=0$.

In the series (5) replace exp $(i x)$ by $V$. The resulting series of operators is absolutely convergent since $\left|V^{n}\right| \leqq K$. Call the transformation which the series defines $A$. That is,

(6) $A=\frac{1}{4 i}\left(V-V^{-1}\right)+\frac{1}{\pi}\left(I-\frac{1}{1 \cdot 3}\left(V^{2}+V^{-2}\right)-\frac{1}{3 \cdot 5}\left(V^{4}+V^{-4}\right)-\cdots\right)$.

Similarly, corresponding to the expansion of $\boldsymbol{g}(x)$, write

$$
B=\frac{1}{4 i}\left(V-V^{-1}\right)-\frac{1}{\pi}\left(I-\frac{1}{1 \cdot 3}\left(V^{2}+V^{-2}\right)-\frac{1}{3 \cdot 5}\left(V^{4}+V^{-4}\right)-\cdots\right) .
$$

The transformation $A \cdot B$ is obtained by multiplying the above series. Since the operations here parallel closely those for evaluating $f(x) \cdot g(x)$, it follows that

$$
A \cdot B=B \cdot A=0 .
$$

Two theorems will now be introduced which will be vital to the following discussion. As their proof has been given by the author elsewhere, only their statement will be reproduced here $\left({ }^{7}\right)$.

Theorem 3. Let $\mathfrak{B}$ be a reflexive vector space. Let $T$ be a bounded linear transformation in $\mathfrak{B}$. Let $\mathfrak{M}$ denote the closed linear manifold of elements $f$ for which $T f=0$; let $\mathfrak{N}$ denote the closed linear manifold spanned by the elements $T f, f \varepsilon \mathfrak{B}$.

(7) Lorch, Means of iterated transformations in reflexive vector spaces, Bulletin of the American Mathematical Society, vol. 45 (1939), pp. 945-947. 
Let $\bar{T}$ be the adjoint of $T$, and let $(\mathfrak{M})$ and $(\mathfrak{N})$ be the manifolds associated with $\bar{T}$ in the manner just indicated. Then $(\mathfrak{M})=\mathfrak{N}^{\perp},(\mathfrak{N})=\mathfrak{M}^{\perp}, \mathfrak{M}=(\mathfrak{N})^{\perp}, \mathfrak{N}=(\mathfrak{M})^{\perp}$.

THEOREM 4. Let $\mathfrak{B}$ be a reflexive vector space. Let $W$ be a bounded linear transformation in $\mathfrak{B}$ such that $\left|W^{n}\right| \leqq K, n=0,1,2, \ldots$. Then the sequence $\left\{T_{n}\right\}$ where $T_{n}=(1 / n) \sum_{s=0}^{n-1} W^{s}$ converges strongly to a limiting transformation $P$ which is a projection. The relation $P f=f$ holds precisely for those elements $f$ for which $W f=f$. The relation $P f=0$ holds precisely for the elements of the closed linear manifold spanned by elements of the form $W g-g, g$ arbitrary in $\mathfrak{B}$. The bound of $P$ satisfies the inequality $|P| \leqq K$.

It is to be noted that if $\mathfrak{M}$ is a closed linear manifold (for short, c.l.m.) in $\mathfrak{B}$, then $\mathfrak{M}^{\perp}$ denotes the c.l.m. of elements of ( $\left.\mathfrak{B}\right)$, each of which is orthogonal to every element of $\mathfrak{M}$. Also to be noted is the fact that the transformation $W$ of Theorem 4 is not required to be bicontinuous.

Slight changes in the hypotheses of Theorem 4 lead to a result which is useful later. The result is valid in any Banach space.

THEOREM $4^{\prime}$. Let $W$ be a transformation in an arbitrary Banach space $\mathfrak{B}$ such that the norm of $W^{n}$ satisfies the inequality $\left|W^{n}\right| \leqq a^{n} K, n=1,2,3, \cdots$, with $a<1, K \geqq 0$. Then $W-I$ possesses a bounded inverse $(W-I)^{-1}$.

A simple proof, based on the Neumann development, may be given. First, if $f \boldsymbol{\varepsilon} \mathfrak{B}$, there exists an element $g$ such that $f=g-W g$. For let

$$
f+W f+W^{2} f+\cdots=g .
$$

The convergence of the series is assured since, for a given $\epsilon>0$,

$$
\begin{aligned}
\left\|W^{n} f+W^{n+1} f+\cdots+W^{m} f\right\| & \leqq\left\|W^{n} f\right\|+\cdots+\left\|W^{m} f\right\| \\
& \leqq K\|f\|\left(a^{n}+\cdots+a^{m}\right)<\epsilon
\end{aligned}
$$

providing $n$ is sufficiently large. Thus

$$
W f+W^{2} f+\cdots=W g
$$

and $f=g-W g$. Hence the range of $W-I$ is $\mathfrak{B}$. Further, if $(W-I) h=0$, then $W h=h, W^{n} h=h$, and $h=0$ since $\left|W^{n}\right| \leqq a^{n} K$. Thus $W-I$ maps $\mathfrak{B}$ into itself in a one-to-one way and is continuous (in one direction). Hence $W-I$ possesses a bounded inverse $\left({ }^{8}\right)$.

Now let $\mathfrak{B}$ be reflexive once more and $V$ be w.a.p. Applying Theorem 4 to the transformation $V^{2}$ (which is uniformly bounded), it is seen that the space $\mathfrak{B}$ is the "sum" of two disjoint $\left({ }^{9}\right)$ manifolds, $\mathfrak{B}=\mathfrak{M}+\mathfrak{N}$ with $V^{2} f=f$ for

(8) Banach, p. 41.

(9) Disjointness is treated in the author's paper On a calculus of operators $\cdots$, p. 220. For short, one may say that two c.l.m.'s $\mathfrak{M}$ and $\mathfrak{R}$ are disjoint if they have in common only the element 0 and if the totality of elements $f+g$ with $f \varepsilon \mathfrak{M}$ and $g \varepsilon \mathfrak{R}$ is a c.l.m. 
every $f \boldsymbol{\varepsilon} \mathfrak{M}$ and with $\mathfrak{N}$ spanned by the elements $\left(V^{2}-I\right) g, g$ arbitrary, or, what amounts to the same, by the elements $\left(V-V^{-1}\right) g$. The reader is reminded that the c.l.m. spanned by a set of elements is the smallest c.l.m. containing the set. For any $f \varepsilon \mathfrak{M}$ write

$$
f=f_{1}+f_{2}, \quad f_{1}=\frac{f+V f}{2}, \quad f_{2}=\frac{f-V f}{2} ;
$$

note that $V f_{1}=f_{1} ; V f_{2}=-f_{2}$. Thus if $\mathfrak{M}^{\prime}$ denotes the c.l.m. of elements $f_{1}$ and $\mathfrak{M}^{\prime \prime}$ denotes the c.l.m. of elements $f_{2}, \mathfrak{M}=\mathfrak{M}^{\prime}+\mathfrak{M}^{\prime \prime}$, the latter manifolds being disjoint. Thus if $f$ is now arbitrary in $\mathfrak{B}$, the decomposition $f=g_{1}+g_{2}+h$ with $g_{1} \varepsilon \mathfrak{M}^{\prime}, g_{2} \varepsilon \mathfrak{M}^{\prime \prime}, h \varepsilon \mathfrak{N}$ is possible and unique. This fact will be of the greatest utility subsequently.

The hypotheses of the following theorem may always be fulfilled for any w.a.p. $V$ by considering the transformation in the c.l.m. $\mathfrak{N}$. The theorem gives the fundamental partition of $\mathfrak{B}$ whose subsequent refinements yield the key to the structure of $V$.

THEOREM 5. Let $V$ be a weakly almost-periodic transformation in a reflexive space $\mathfrak{B}$. Let the equation $V^{2} f=f$ have the unique solution $f=0$. Let $A$ and $B$ be the linear transformations introduced in (6) and (7) respectively. Let $\mathfrak{Q}$ be the closed linear manifold of elements $f$ for which $A f=0$; let $\Re$ be the closed linear manifold of elements $f$ for which $B f=0$. Then $\mathfrak{Q}$ and $\Re$ have only the element 0 in common; together they span $\mathfrak{B}$. The manifold pair $\{\mathfrak{Q}, \mathfrak{R}\}$ reduces $V$ in the sense that if $g \in \mathfrak{E}, \operatorname{Vg} \varepsilon \mathfrak{Q} ;$ if $h \in \mathcal{R}, V h \varepsilon \Re$.

Since $A \cdot B=B \cdot A=0$ by (8), the c.l.m. $\mathfrak{Q}^{\prime}$ spanned by the elements $B f$, $f \boldsymbol{\varepsilon} \mathfrak{B}$ is included in $\mathfrak{O}$. Similarly, the c.l.m. $\mathfrak{R}^{\prime}$ spanned by the elements $A f$ is included in $\Re$.

Applying Theorem 4, we see that since $\left(V^{2}-I\right) f=0$ implies $f=0$, the space $\mathfrak{B}$ is spanned by the elements of form $\left(V-V^{-1}\right) f, f \varepsilon \mathfrak{B}$. Hence the elements $(A+B) f, f \boldsymbol{\varepsilon} \mathfrak{B}$. Since $A f \boldsymbol{\varepsilon} \Re, B f \boldsymbol{\varepsilon} \mathfrak{O}$, the manifolds $\mathfrak{Q}$ and $\mathfrak{R}$ span $\mathfrak{B}$.

If $f \varepsilon \mathfrak{Q}, f \varepsilon \Re$, then $A f=B f=0$ or $(A+B) f=0$. Hence $f=0$; thus $\mathfrak{Q}$ and $\Re$ have only the element 0 in common.

Since $A V=V A$ and $B V=V B$ (see (6) and (7)), then $A g=0$ implies $A V g=0 ; B h=0$ implies $B V h=0$. Thus the manifold pair $\{\mathfrak{Q}, \Re\}$ reduces $V$ in the sense explained above.

It may quickly be seen that the manifolds $\mathfrak{Q}^{\prime}$ and $\Re^{\prime}$ have all the properties which the theorem ascribes to $\mathfrak{Q}$ and $\Re$. In case $\mathfrak{Q}^{\prime}$ and $\Re^{\prime}$ are disjoint, $\mathfrak{Q}=\mathfrak{Q}^{\prime}$ and $\Re=\Re^{\prime}$. For if $\mathfrak{Q}^{\prime}$ and $\Re^{\prime}$ are disjoint, for an arbitrary $f \boldsymbol{\varepsilon} \mathfrak{B}$ one may find a $g \varepsilon \mathfrak{Q}^{\prime}$ and an $h \varepsilon \Re^{\prime}$ such that $f=g+h$. If now in particular $f \varepsilon \mathfrak{Q}$, then $f-g=h \varepsilon \mathfrak{O}$ as well as $h \varepsilon \Re^{\prime}$. Since $\Re \supset \Re^{\prime}, h=0$ and $f \varepsilon \mathfrak{Q}^{\prime}$. Thus $\mathfrak{Q}=\mathfrak{Q}^{\prime}$; similarly $\Re=\Re^{\prime}\left({ }^{10}\right)$.

(10) If the c.l.m.'s $\mathfrak{Q}$ and $\Re$ are not disjoint, it is easy to construct a c.l.m. $\subseteq$ of which $\mathfrak{Q}$ is a proper subset and such that $\Re$ and $\subseteq$ have only the element 0 in common. For let $f$ be any 
In the special case in which $V$ is a unitary transformation in a Hilbert space, $\mathfrak{Q}$ and $\Re$ may be shown to be orthogonal in the following way. Using the fact that $\bar{V}=V^{-1}$, it may be seen by direct computation in (6) that $A=\bar{A}$ ( $A$ is self-adjoint). Thus the c.l.m. spanned by the elements $\bar{A} f$ is $\Re^{\prime}$. By Theorem 3 , this c.l.m. is the orthogonal complement of $\mathfrak{Q}$. Thus $\mathfrak{Q}^{\prime}$ and $\Re^{\prime}$ are orthogonal to each other, hence disjoint. By the previous paragraph $\mathfrak{Q}=\mathfrak{Q}$, $\Re=\Re^{\prime}$.

Attempts to determine whether the manifolds $\mathfrak{Q}$ and $\Re$ are disjoint in general have not been successful. The following theorem gives some criteria which may be useful.

THEOREM 6. Let $V$ be a weakly almost-periodic transformation satisfying the conditions given in Theorem 5. The closed linear manifolds $\mathfrak{Q}$ and $\Re$ there defined are disjoint if either of the following conditions is satisfied:

(a) The transformation $A+B$ possesses a bounded inverse $(A+B)^{-1}$.

(b) The transformation $I-A$ (or $I-B)$ is uniformly bounded, $\left|(I-A)^{n}\right|$ $\leqq L, n=1,2, \cdots$.

If $(A+B)^{-1}$ exists and is bounded, then for any $f \varepsilon \mathfrak{B}$,

$$
f=(A+B)(A+B)^{-1} f=A(A+B)^{-1} f+B(A+B)^{-1} f
$$

which gives the resolution of $f$ into two elements, the first in $\Re$, the second in $\mathfrak{Q}$. Since $\mathfrak{Q}$ and $\Re$ have only the element 0 in common, this resolution is unique. Thus $\mathfrak{O}$ and $\Re$ are disjoint.

Suppose that the transformation $I-A$ is uniformly bounded, $\left|(I-A)^{n}\right|$ $\leqq L, n=1,2, \cdots$. Then by Theorem 4 , the two following manifolds are disjoint: the c.l.m. of elements $f$ for which $(I-A) f=f$, that is, the c.l.m. $\mathfrak{Q}$; and the c.l.m. spanned by the elements $[(I-A)-I] f=-A f$, that is, the c.l.m. $\Re^{\prime}$. Thus $\mathfrak{Q}^{\prime}$ and $\Re^{\prime}$ are disjoint and $\mathfrak{Q}=\mathfrak{Q}^{\prime} \Re=\Re^{\prime}$.

In this connection, it may be pointed out that the uniform boundedness of the transformations $A+B, A$, and $B$ may be proved easily. Since $A+B=\left(V-V^{-1}\right) / 2 i$, it may be seen by direct multiplication that $\left|(A+B)^{n}\right|$ $\leqq K$ where $K$ is a uniform bound for $V^{n}$. Since $A^{n}=A(A+B)^{n-1}$ and $B^{n}=B(A+B)^{n-1}, A$ and $B$ are uniformly bounded. Similarly the uniform boundedness of the transformation $I-(A+B)^{2}$ may be demonstrated. Also demonstrable is the uniform boundedness on the c.l.m. $\mathfrak{Q}^{\prime}$ or on the c.l.m. $\Re^{\prime}$ of the transformation $I-A^{2}$.

If the manifolds $\mathfrak{Q}$ and $\Re$ are disjoint and if certain even more stringent conditions involving uniformity of bound are satisfied, it is possible to con-

element which cannot be represented in the form $g+h$ with $g \varepsilon \mathfrak{Q}, h \varepsilon \Re$. Then the totality $\mathbb{S}$ of elements of the form $\alpha f+g, g \varepsilon \mathfrak{Q}$ is a c.l.m. having the required properties. That $\subseteq$ is closed may be seen as follows: Let $\left\{\alpha_{n} f+g_{n}\right\}$ be a convergent sequence of elements in $\Im$. Let $F$ be a linear functional which is orthogonal to $\mathfrak{Q}$ and for which $F f=1$. Then $F\left(\alpha_{n} f+g_{n}\right)=\alpha_{n} \rightarrow \alpha$. Hence $g_{n} \rightarrow g \varepsilon \mathfrak{Q}$ and $\alpha_{n} f+g_{n} \rightarrow \alpha f+g \varepsilon \widetilde{S}$. 
struct for $V$ a resolution of the identity $\left({ }^{11}\right)$. The present situation, however, leads to a structure much more general it seems than that of a resolution of the identity. It is described in the next theorem. This theorem completes the analysis or decomposition of $V$; the reconstruction of $V$ from the manifold pairs which reduce it will be undertaken subsequently.

A useful lemma will first be established. Let $\lambda$ be so chosen that $-\pi<\lambda \leqq \pi$. The transformation $V_{\lambda}=\exp (-i \lambda) V$ is w.a.p. In what follows, $A_{\lambda}$ and $B_{\lambda}$ will denote the transformations which are constructed from $V_{\lambda}$ in the manner in which $A=A_{0}$ and $B=B_{0}$ are constructed from $V=V_{0}$.

Lemma. Let $\lambda_{1}, \lambda_{2}, \cdots, \lambda_{n}$ be any distinct real numbers with $-\pi<\lambda_{j} \leqq 0$. Then for any w.a.p. transformation $V$ and for every $f \in \mathfrak{B}$, the following decomposition is possible and unique:

$$
f=\sum_{j=1}^{n}\left(g_{\lambda_{j}}+g_{\lambda_{j}}^{\prime}\right)+h
$$

with

$$
V g_{\lambda_{j}}=e^{i \lambda_{j}} g_{\lambda_{j}}, \quad V g_{\lambda_{j}}^{\prime}=-e^{i \lambda_{j}} g_{\lambda_{j}}^{\prime},
$$

and with $h$ in the closed linear manifold spanned by the elements of the form

$$
X k=\left(A_{\lambda_{1}}+B_{\lambda_{1}}\right)\left(A_{\lambda_{2}}+B_{\lambda_{2}}\right) \cdots\left(A_{\lambda_{n}}+B_{\lambda_{n}}\right) k,
$$

$k$ arbitrary in $\mathfrak{B}$.

Let the projection which Theorem 4 defines for $V_{\lambda}$ be denoted by $P_{\lambda}$; then $V_{\lambda} P_{\lambda} f=P_{\lambda} f$ or $V P_{\lambda} f=\exp (i \lambda) P_{\lambda} f$. Let the projection which is defined for $-V_{\lambda}=V_{\lambda+\pi}$ be denoted by $P_{\lambda}^{\prime}$; then $V P_{\lambda}^{\prime} f=-\exp (i \lambda) P_{\lambda}^{\prime} f$. Note that the projections of the collection $P_{\lambda}$ and $P_{\lambda}^{\prime}$ for all $\lambda,-\pi<\lambda \leqq 0$, are commutative. Indeed, the product of two distinct projections is 0 . Hence

$$
\begin{aligned}
I & =\prod_{j=1}^{n}\left[P_{\lambda_{j}}+\left(I-P_{\lambda_{j}}\right)\right]\left[P_{\lambda_{j}}^{\prime}+\left(I-P_{\lambda_{j}}^{\prime}\right)\right] \\
& =\sum_{j=1}^{n}\left(P_{\lambda_{j}}+P_{\lambda_{j}}^{\prime}\right)+\prod_{j=1}^{n}\left(I-P_{\lambda_{j}}\right)\left(I-P_{\lambda_{j}}^{\prime}\right) .
\end{aligned}
$$

This gives a resolution for $f \boldsymbol{\varepsilon} \mathfrak{B}$ of the type indicated in the lemma except for the fact that the announced properties of $h$ have not yet been established. To prove that the resolution is unique let $\sum_{j=1}^{n}\left(g_{\lambda_{j}}+g_{\lambda_{j}}^{\prime}\right)+h=0$; operating with $P_{\lambda_{r}}$, one obtains $P_{\lambda_{r}} g_{\lambda_{r}}=g_{\lambda_{r}}=0$ for $r=1,2, \cdots, n$. Similarly $g_{\lambda_{r}}^{\prime}=0$, $r=1, \cdots, n$, hence finally $h=0$. The resolution is thus unique.

It remains to be shown that $h$ has the character specified in the lemma. Since $h$ is of the form $\left(I-P_{\lambda_{j}}\right) k$, by Theorem $4 h$ is in the c.l.m. spanned by the elements of the form $\left(V_{\lambda_{j}}-I\right) l$; similarly, it is in the c.l.m. spanned by the ele-

(11) Lorch, On a calculus $\cdots$, p. 226, Definition 4. 
ments of the form $\left(-V_{\lambda_{j}}-I\right) l$. Now the intersection of these two manifolds includes the c.l.m. spanned by the elements of the form $\left(A_{\lambda_{j}}+B_{\lambda_{j}}\right) l$. If $Y=\prod_{j=1}^{n}\left(I-P_{\lambda_{j}}\right)\left(I-P_{\lambda_{j}}^{\prime}\right)$, then $Y$ is a projection and the c.l.m. of elements $Y l$ is precisely the intersection of the c.l.m.'s spanned by the elements $\left(V_{\lambda_{j}}-I\right) l$ and $\left(-V_{\lambda_{j}}-I\right) l, j=1, \cdots, n$. Hence the c.l.m. of all elements $Y l$ includes the c.l.m. spanned by the elements $X l$.

It will be shown that the manifold spanned by the elements $X l$ is identical with the manifold of the elements $Y l$. To do this it is necessary and sufficient to establish that the collection of elements $\sum_{j=1}^{n}\left(g_{\lambda_{j}}+g_{\lambda_{j}}^{\prime}\right)+X l$ is dense in $\mathfrak{B}$. If $f \varepsilon \mathfrak{B}$, then $f$ may be approximated in the norm by elements $g_{\lambda_{1}}+g_{\lambda_{1}}^{\prime}+\left(A_{\lambda_{1}}+B_{\lambda_{1}}\right) h_{1}$ (see discussion preceding Theorem 5). Now $h_{1}$ may be approximated by elements $g_{\lambda_{2}}+g_{\lambda_{2}}^{\prime}+\left(A_{\lambda_{2}}+B_{\lambda_{2}}\right) h_{2}$. Since $A_{\lambda_{1}} g_{\lambda_{2}}=f\left(\lambda_{2}-\lambda_{1}\right) g_{\lambda_{2}}$ (that is, $A_{\lambda_{1}} g_{\lambda_{2}}=\sin \left(\lambda_{2}-\lambda_{1}\right) g_{\lambda_{2}}$ or 0$)$, and $A_{\lambda_{1}} g_{\lambda_{2}}^{\prime}=f\left(\pi+\lambda_{2}-\lambda_{1}\right) g_{\lambda_{2}}^{\prime}$, and since $B_{\lambda_{1}} g_{\lambda_{2}}=\boldsymbol{g}\left(\lambda_{2}-\lambda_{1}\right) g_{\lambda_{2}}, B_{\lambda_{1}} g_{\lambda_{2}}^{\prime}=\boldsymbol{g}\left(\pi+\lambda_{2}-\lambda_{1}\right) g_{\lambda_{2}}^{\prime}, f$ may be approximated by elements of the form $g_{\lambda_{1}}+g_{\lambda_{1}}^{\prime}+\rho g_{\lambda_{2}}+\sigma g_{\lambda_{2}}^{\prime}+\left(A_{\lambda_{1}}+B_{\lambda_{1}}\right)\left(A_{\lambda_{2}}+B_{\lambda_{2}}\right) h_{2}$, where $\rho$ and $\sigma$ are some constants. Now let $h_{2}$ be approximated by an element $g_{\lambda_{3}}+g_{\lambda_{3}}^{\prime}+\left(A_{\lambda_{3}}+B_{\lambda_{3}}\right) h_{3}$, etc. Proceeding in this way, it can be seen that any element $f$ can be approximated by elements of the form $\sum\left(g_{\lambda_{j}}+g_{\lambda_{j}}^{\prime}\right)+X l$.

Note that if $\mathfrak{M}$ is a c.l.m. which has the property that it contains $V f$ whenever it contains $f$, then along with $f, \mathfrak{M}$ contains $g_{\lambda_{j}}, g_{\lambda_{j}}^{\prime}, j=1, \cdots, n$, and $h$. For

$$
g_{\lambda_{j}}=P_{\lambda_{j}} f=\lim _{n \rightarrow \infty} \frac{1}{n} \sum_{s=0}^{n-1} V_{\lambda_{j}}^{s} f
$$

hence $g_{\lambda_{j}} \varepsilon \mathfrak{M}$. Similarly the $g_{\lambda_{j}}^{\prime}$ and thus $h$ are in $\mathfrak{M}$.

If in (9) one operates upon $f$ with $V$, one obtains the decomposition of $V f$, that is, $V f=\sum_{j=1}^{n}\left(V g_{\lambda_{j}}+V g_{\lambda_{j}}^{\prime}\right)+V h$ is the decomposition (9) for the element $V f$. This is because the projections $P_{\lambda_{j}}, P_{\lambda_{j}}^{\prime}$ are commutative with $V$. What is here stated for $V$ applies equally well to any function of $V$, say , $A, B$, etc.

THEOREM 7. Let $V$ be a weakly almost-periodic transformation in a reflexive vector space $\mathfrak{B}$. Then for every real number $\lambda,-\infty<\lambda<\infty$, there exists a pair of closed linear manifolds, $\left\{\mathfrak{F}_{\lambda}, \mathfrak{F}_{\lambda}\right\}$, possessing the following properties:

(a) The pair $\left\{\mathfrak{E}_{\lambda}, \mathfrak{F}_{\lambda}\right\}$ reduces $V$ in the sense of Theorem 5 .

(b) The manifolds $\mathfrak{E}_{\lambda}$ and $\mathfrak{F}_{\lambda}$ have in common only the element 0 ; together, $\mathfrak{F}_{\lambda}$ and $\mathfrak{F}_{\lambda} \operatorname{span} \mathfrak{B}$.

(c) $\mathfrak{E}_{\lambda} \supset \mathbb{F}_{\mu}$ for $\lambda>\mu ; \mathfrak{F}_{\lambda} \subset \mathfrak{F}_{\mu}$ for $\lambda>\mu$.

(d) $\mathfrak{E}_{-\pi}=0, \mathfrak{F}_{-\pi}=\mathfrak{B} ; \mathfrak{E}_{\pi}=\mathfrak{B}, \mathfrak{F}_{\pi}=0$.

Preliminary to constructing the $\mathbb{E}_{\lambda}$ and $\mathfrak{F}_{\lambda}$ certain other manifolds $\mathfrak{M}_{\lambda}$ and $\mathfrak{N}_{\lambda},-\pi<\lambda \leqq \pi$, will be introduced. For $-\pi<\lambda \leqq 0$, apply the lemma just established to the case $n=1, \lambda_{1}=\lambda$; thus $f=g_{\lambda}+g_{\lambda}^{\prime}+h$ with $V g_{\lambda}=\exp (i \lambda) g_{\lambda}$, $V g_{\lambda}{ }^{\prime}=-\exp (i \lambda) g_{\lambda}{ }^{\prime}$, and with $h$ in the c.l.m. spanned by the elements $\left(A_{\lambda}+B_{\lambda}\right) l$. Let $\mathfrak{M}_{\lambda}$ be the collection of all elements $f$ such that $A_{\lambda} f=0$ and 
such that $g_{\lambda}^{\prime}=0$. Let $\mathfrak{R}_{\lambda}$ be the collection of all elements $f$ such that $B_{\lambda} f=0$ and such that $g_{\lambda}=0$. In the first case, from $A_{\lambda} f=0$ follows $A_{\lambda} h=0$. Thus $\mathfrak{M}_{\lambda}$ is the c.l.m. which is the direct sum of the following two disjoint manifolds: The c.l.m. of all elements $g_{\lambda}$ for which $V g_{\lambda}=\exp (i \lambda) g_{\lambda}$; the c.l.m. of all elements $h$ for which $A_{\lambda} h=0$. Similarly, $\mathfrak{N}_{\lambda}$ is the c.l.m. which is the direct sum of the two disjoint manifolds: The c.l.m. of all elements $g_{\lambda}^{\prime}$ for which $V g_{\lambda}{ }^{\prime}=-\exp (i \lambda) g_{\lambda}{ }^{\prime}$; the c.l.m. of all elements $h$ for which $B_{\lambda} h=0$.

For $0<\lambda \leqq \pi$, let $\mathfrak{M}_{\lambda}$ and $\mathfrak{N}_{\lambda}$ be defined by $\mathfrak{M}_{\lambda}=\mathfrak{N}_{\lambda-\pi}$, $\mathfrak{N}_{\lambda}=\mathfrak{M}_{\lambda-\pi}$. Since $A_{\lambda}=-B_{\lambda-\pi}, B_{\lambda}=-A_{\lambda-\pi}, \mathfrak{M}_{\lambda}$ is precisely the c.l.m. which is the direct sum of two c.l.m.'s: the c.l.m. of the elements $g$ for which $V g=\exp (i \lambda) g$; and the c.l.m. of the elements $h$ for which $A_{\lambda} h=0$. A similar statement may be made for $\mathfrak{N}_{\lambda}$.

The pair $\left\{\mathfrak{M}_{\lambda}, \mathfrak{N}_{\lambda}\right\}$ reduces $V$. Assume $-\pi<\lambda \leqq 0$. If $f \varepsilon \mathfrak{M}_{\lambda}, f=g_{\lambda}+h$, $V f=V g_{\lambda}+V h, A_{\lambda} V f=V A_{\lambda} f=0$, hence $V f \varepsilon \mathfrak{M}_{\lambda}$. Similarly for $\mathfrak{N}_{\lambda}$.

The manifolds $\mathfrak{M}_{\lambda}$ and $\mathfrak{N}_{\lambda}$ have only the element 0 in common. Let $f \boldsymbol{\varepsilon} \mathfrak{M}_{\lambda}$, $f \varepsilon \mathfrak{N}_{\lambda}, f=g_{\lambda}+h, f=g_{\lambda}{ }^{\prime}+k$. Then $0=g_{\lambda}-g_{\lambda}{ }^{\prime}+h-k, g_{\lambda}=g_{\lambda}{ }^{\prime}=0, f=h=k$ and $A_{\lambda} h=B_{\lambda} h=\left(A_{\lambda}+B_{\lambda}\right) h=0$; thus $h=0$.

The manifolds $\mathfrak{M}_{\lambda}$ and $\mathfrak{N}_{\lambda}$ span $\mathfrak{B}$. For the elements $g_{\lambda}+g_{\lambda}{ }^{\prime}+\left(A_{\lambda}+B_{\lambda}\right) k$ lie dense in $\mathfrak{B}$; all elements $g_{\lambda}$ and $B_{\lambda} k$ lie in $\mathfrak{M}_{\lambda}$; the elements $g_{\lambda}{ }^{\prime}$ and $A_{\lambda} k$ lie in $\mathfrak{N}_{\lambda}$.

The manifolds $\mathfrak{E}_{\lambda}, \mathfrak{F}_{\lambda},-\infty<\lambda<\infty$, will be defined. For $-\infty<\lambda \leqq-\pi$, $\mathfrak{E}_{\lambda}=0$ and $\mathfrak{F}_{\lambda}=\mathfrak{B}$. For $\pi \leqq \lambda<\infty, \mathfrak{E}_{\lambda}=\mathfrak{B}$ and $\mathfrak{F}_{\lambda}=0$. In discussing the theorem, these values of $\lambda$ will receive no attention.

Let $-\pi<\lambda<0$. Consider the partition for $f \varepsilon \mathfrak{B}$ of the lemma for $n=2$, $\lambda_{1}=0, \lambda_{2}=\lambda$ :

$$
f=g_{0}+g_{0}^{\prime}+g_{\lambda}+g_{\lambda}{ }^{\prime}+h .
$$

The c.l.m. $\mathbb{E}_{\lambda}$ is defined to be the collection of elements $f$ for which $A f=A_{\lambda} f=0$, and such that $g_{0}=g_{0}^{\prime}=g_{\lambda}^{\prime}=0$. The c.l.m. $\mathfrak{F}_{\lambda}$ is defined to be the collection of elements $f$ for which $B B_{\lambda} f=0$ and such that $g_{\lambda}=0$.

If $0<\lambda<\pi$, write $\mu=\lambda-\pi$ and consider the partition for $f$ of the lemma corresponding to $n=2, \lambda_{1}=0, \lambda_{2}=\mu$. Then $E_{\lambda}$ is defined to be the c.lm. of elements $f$ for which $A B_{\mu} f=-A A_{\lambda} f=0$ and such that $g_{0}^{\prime}=0$. Also, $F_{\lambda}$ is defined to be c.l.m. of elements $f$ for which $B f=0, A_{\mu} f=0$ (note that $A_{\mu}=-B_{\lambda}$ ), and such that $g_{0}=g_{\mu}=g_{\mu}^{\prime}=0$.

For $\lambda=0, \mathfrak{E}_{0}=\mathfrak{M}_{0}$ and $\mathfrak{F}_{0}=\mathfrak{R}_{0}$ by definition.

It may be noted that for $-\pi<\lambda \leqq 0, \mathbb{E}_{\lambda}$ is precisely the intersection of $\mathfrak{M}_{0}$ and $\mathfrak{M}_{\lambda}$; and $\mathfrak{F}_{\lambda}$ contains $\mathfrak{R}_{0}$ and $\mathfrak{N}_{\lambda}$.

Toward (a). Let $-\pi<\lambda<0$. As stated above, the partition (9) for $V f$ is obtained from that of $f$ by operating upon (9) with $V$. Since $A V=V A$, and $A_{\lambda} V=V A_{\lambda}$, whenever $f \varepsilon \mathcal{E}_{\lambda}$, then $V f \varepsilon \mathcal{E}_{\lambda}$. Similarly since $V B B_{\lambda}=B B_{\lambda} V$, if $f \varepsilon \mathfrak{F}_{\lambda}$, then $V f \boldsymbol{\varepsilon} \mathfrak{F}_{\lambda}$. 
Toward (b). Suppose $f \varepsilon \mathcal{E}_{\lambda}, f=g_{\lambda}+h$; if $f \varepsilon \mathfrak{F}_{\lambda}$, then $f=g_{0}+g_{0}^{\prime}+g_{\lambda}^{\prime}+k$. Thus $0=g_{0}+g_{0}^{\prime}-g_{\lambda}+g_{\lambda}^{\prime}+k-h$ and $g_{0}=g_{0}^{\prime}=g_{\lambda}=g_{\lambda}^{\prime}=0, f=k=h$. Further, since $A h=A_{\lambda} h=B B_{\lambda} h=0,(A+B)\left(A_{\lambda}+B_{\lambda}\right) h=0$ and $h=0$. Thus $\mathfrak{F}_{\lambda}$ and $\mathfrak{F}_{\lambda}$ have only the element 0 in common. To prove that $\mathscr{F}_{\lambda}$ and $\mathfrak{F}_{\lambda}$ span $\mathfrak{B}$, note that $g_{\lambda} \varepsilon \mathfrak{F}_{\lambda}, g_{0}, g_{0}^{\prime}, g_{\lambda}^{\prime} \varepsilon \mathfrak{F}_{\lambda}$, and also that $h$ may be approximated by elements of the form $(A+B)\left(A_{\lambda}+B_{\lambda}\right) k$ with $B B_{\lambda} k$ in $\xi_{\lambda}$ and with $A A_{\lambda} k, B A_{\lambda} k$, $A B_{\lambda} k$ in $\mathfrak{F}_{\lambda}$.

Toward (c). Let $-\pi<\lambda<\mu<0$. Let $f \varepsilon \xi_{\lambda}$; it will be shown that $f \varepsilon \xi_{\mu}$. Note that $B B_{\lambda} A_{\mu}=0$ identically in $\mathfrak{B}$. This may be seen as follows: The transformation $B$ is constructed with the help of the function $g(x)$-see (5), (6), and (7). Similarly, the transformations $A_{\mu}$ and $B_{\lambda}$ are constructed with the help of the functions $f(x-\mu)$ and $g(x-\lambda)$ respectively. Now $\boldsymbol{g}(x) \cdot \boldsymbol{g}(x-\lambda) \cdot \boldsymbol{f}(x-\mu)=0$ identically in $x$. This product may be evaluated by multiplying together three Fourier series, yielding a product series having zero coefficients. Precisely the same series operations occur in constructing the transformation $B B_{\lambda} A_{\mu}$ except that $\exp (i x)$ is replaced by $V$. Thus $B B_{\lambda} A_{\mu}=0$. Since $f \varepsilon \mathcal{E}_{\lambda}, A f=A_{\lambda} f=0$ and

$$
(A+B)\left(A_{\lambda}+B_{\lambda}\right) A_{\mu} f=B B_{\lambda} A_{\mu} f=0 .
$$

Let $f=g_{\lambda}+h$ be the decomposition (9) of $f$ for $n=2, \lambda_{1}=0, \lambda_{2}=\lambda$. Then $A_{\mu} f=A_{\mu} g_{\lambda}+A_{\mu} h=A_{\mu} h$ since $A_{\mu} g_{\lambda}=\boldsymbol{f}(\lambda-\mu) g_{\lambda}=0$. Also $(A+B)\left(A_{\lambda}+B_{\lambda}\right) A_{\mu} h=0$ implies $A_{\mu} h=0$, that is, $A_{\mu} f=0$. Let $f=g_{0}+g_{0}^{\prime}+g_{\mu}+g_{\mu}^{\prime}+k$ be the decomposition (9) of $f$ for $n=2, \lambda_{1}=0, \lambda_{2}=\mu$. Since $f \varepsilon \mathcal{E}_{\lambda}, g_{0}=g_{0}^{\prime}=0$. Further since $A f=0, A g_{\mu}^{\prime}=\sin (\mu+\pi) g_{\mu}^{\prime}=0$, and $g_{\mu}^{\prime}=0$. Therefore $f \varepsilon \xi_{\mu}$ and $\mathbb{E}_{\lambda} \subset \xi_{\mu}$.

It will now be proved that $\mathfrak{F}_{\mu} \subset \mathfrak{F}_{\lambda}$ (for $-\pi<\lambda<\mu<0$ ). Let $f \varepsilon \mathfrak{F}_{\mu}$; then $B B_{\mu} f=0$. Also,

$$
\left(A_{\mu}+B_{\mu}\right) B B_{\lambda} f=B_{\mu} B B_{\lambda} f=0
$$

since $A_{\mu} B B_{\lambda}=0$. Let $f=g_{0}+g_{0}^{\prime}+g_{\mu}+g_{\mu}^{\prime}+h$ be the decomposition (9) for $n=2$, $\lambda_{1}=0, \lambda_{2}=\mu$. Since $f \varepsilon \mathfrak{F}_{\mu}, g_{\mu}=0$. Thus $B B_{\lambda} f=B B_{\lambda} g_{\mu}^{\prime}+B B_{\lambda} h=B B_{\lambda} h$ (since $\left.B g_{\mu}^{\prime}=\boldsymbol{g}(\mu+\pi) g_{\mu}^{\prime}=0\right)$ is the decomposition (9) of $B B_{\lambda} f$. Applying the operator $\left(A_{\mu}+B_{\mu}\right)$, one sees that $B B_{\lambda} f=0$. Now let $f=g_{0}+g_{0}^{\prime}+g_{\lambda}+g_{\lambda}^{\prime}+h$ be the partition of $f$ relative to $n=2, \lambda_{1}=0, \lambda_{2}=\lambda$. Since $B B_{\mu} f=0, B B_{\mu} g_{\lambda}=0$, that is, $\boldsymbol{g}(\lambda) \cdot \boldsymbol{g}(\lambda-\mu) g_{\lambda}=0$, or $g_{\lambda}=0$. Hence $f \boldsymbol{\varepsilon} \mathfrak{F}_{\lambda}$, and $\mathfrak{F}_{\mu} \subset \mathfrak{F}_{\lambda}$.

The method of proof of the properties (b) and (c) for other values (and pairs of values) of $\lambda$, for instance for $0<\lambda<\pi$, is now clear and will not be set down here. The proof of (d) lies in the definition of $\mathfrak{E}_{\lambda}$ and $\mathfrak{F}_{\lambda}$ for $\lambda=-\pi, \pi$.

\section{The integral Representation of $V$}

The aim of this chapter is to establish an integral representation (2) for the w.a.p. transformation $V$. The precise interpretation of (2) in the present case will be given in the next theorem. Preparatory to this theorem, a few auxiliary devices will be investigated. 
Let $\epsilon_{2}>0$ be any positive number. Suppose that for $f \varepsilon \mathfrak{B},(V-I)^{2} f=\eta$ with $\|\eta\| \leqq \epsilon_{1}\|f\|$ for some $\epsilon_{1}>0$. Then

$$
\begin{aligned}
&\left(V^{2}-V\right) f=(V-I) f+\eta, \\
&\left(V^{3}-V^{2}\right) f=(V-I) f+V \eta+\eta, \\
& \text {. . . . . . . . . . . . . . . . . . } \\
&\left(V^{n+1}-V^{n}\right) f=(V-I) f+V^{n-1} \eta+\cdots+V \eta+\eta .
\end{aligned}
$$

Thus

$$
n(V-I) f=\left(V^{n+1}-V\right) f-n \eta-(n-1) V \eta-\cdots-V^{n-1} \eta
$$

or

$$
\|(V-I) f\| \leqq \frac{2}{n} K\|f\|+\frac{n+1}{2} K \epsilon_{1}\|f\|
$$

If now $n$ is chosen so large that $2 K / n \leqq \epsilon_{2} / 2$, and if it happens for that $n$ that $(n+1) K \epsilon_{1} \leqq \epsilon_{2}$, then $\|(V-I) f\| \leqq \epsilon_{2}\|f\|$.

Suppose now that for some $f \varepsilon \mathfrak{B},\left(V^{2}-I\right) f=\eta$ with $\|\eta\| \leqq \epsilon\|f\|$ for some $\epsilon>0$. Then writing $f=f_{1}+f_{2}$ with

$$
f_{1}=\frac{f+V f}{2}, \quad f_{2}=\frac{f-V f}{2},
$$

it is seen that $(V-I) f_{1}=\eta / 2,(V+I) f_{2}=-\eta / 2$. Thus it follows if $\epsilon$ is "small" that $f$ is the sum of two elements for which $V$ is "almost" the identity transformation or its negative. The following theorem indicates precisely in what sense the transformation $V$ may be reconstructed from $\left\{\mathbb{E}_{\lambda}, \mathfrak{F}_{\lambda}\right\}$.

THEOREM 8. Let a real number $\lambda$ and an $\epsilon>0$ be given. Then it is possible to find a closed linear manifold $\mathfrak{A}_{\lambda}$ such that:

(a) The manifold $\mathfrak{A}_{\lambda}$ lies in $\mathfrak{E}_{\lambda+\epsilon}$ and $\mathfrak{F}_{\lambda-\epsilon}$.

(b) If $f \varepsilon \mathfrak{U}_{\lambda}$, the inequality

$$
\left\|\left(V-e^{i \lambda} I\right) f\right\| \leqq \epsilon\|f\|
$$

holds.

Furthermore there exists a finite collection of values $\lambda$, viz., $\lambda_{1}, \lambda_{2}, \cdots, \lambda_{n}$, such that the space $\mathfrak{B}$ is spanned by the manifolds $\mathfrak{A}_{\lambda_{i}}, i=1,2, \cdots, n$.

Consider the transformation $A_{\lambda-\delta} \cdot B_{\lambda+\delta}$, with $\lambda$ arbitrary but fixed, $\delta>0$ arbitrary. Its bound is a continuous function of $\delta$ which is zero for $\delta=0$. Writing $\lambda-\delta=\alpha, \lambda+\delta=\beta$,

$$
A_{\alpha} B_{\beta} \cdot A_{\alpha} B_{\beta}=\left(A_{\alpha}+B_{\alpha}\right)\left(A_{\beta}+B_{\beta}\right) A_{\alpha} B_{\beta} .
$$

By choosing $\delta$ sufficiently small, the norm of 


$$
\left(A_{\lambda}+B_{\lambda}\right)^{2}-\left(A_{\alpha}+B_{\alpha}\right)\left(A_{\beta}+B_{\beta}\right)
$$

may be made less than $\epsilon_{1} / 2$ where $\epsilon_{1}>0$ is arbitrary. This choice of $\delta$ is independent of $\lambda$. If at the same time $\delta$ is so small that $\left|A_{\alpha} B_{\beta}\right| \leqq \epsilon_{1} / 2$, then

$$
\left\|\left(A_{\lambda}+B_{\beta}\right)^{2} A_{\alpha} B_{\lambda} f\right\| \leqq \epsilon_{1}\left\|A_{\alpha} B_{\beta} f\right\| .
$$

Once more, this choice of $\delta$ is uniform for all $\lambda$. Since

$$
A_{\lambda}+B_{\lambda}=(1 / 2 i)\left(\exp (-i \lambda) V-\exp (i \lambda) V^{-1}\right),
$$

it is seen that, for $g=A_{\alpha} B_{\beta} f,\left\|\left(V^{2}-\exp (2 i \lambda)\right)^{2} g\right\| \leqq 4 K \epsilon_{1}\|g\|$. This inequality holds not only for the elements $g$ but for all elements in the c.l.m. $\mho_{\lambda \delta}$ spanned by the $g$.

Applying (10) and what follows, it is possible to write for all $g \varepsilon \widetilde{夭}_{\lambda \delta}$

$$
\left\|\left(V^{2}-e^{2 i \lambda}\right) g\right\| \leqq \epsilon_{2}\|g\|
$$

provided that $4 K^{2}(n+1) \epsilon_{1} \leqq \epsilon_{2}$ where the integer $n$ is properly chosen as indicated above.

Let $g=g_{1}+g_{2}$ where the resolution is made as in (11) with $V$ replaced by $\exp (-i \lambda) V$. Writing $(V+\exp (i \lambda)) g_{2}=\zeta$, it is clear that $\|\zeta\| \leqq \epsilon_{2}\|g\| / 2$. Henceforth assume that $\delta<\pi / 2$. Simple considerations show that, for such a $\delta$, $A_{\lambda-\delta} B_{\lambda+\delta} B_{\lambda-\pi / 2}=0$ identically in $\mathfrak{B}$. Writing $B_{\lambda}=\sum_{-\infty}^{\infty} \alpha_{s}(\lambda) V^{s}$, it is seen that, for a given $\epsilon_{3}>0$, an integer $r \geqq 1$ may be found such that

$$
K \sum_{r}^{\infty}\left|\alpha_{8}(\lambda)\right|+K \sum_{-\infty}^{-r}\left|\alpha_{8}(\lambda)\right| \leqq \epsilon_{3}
$$

for all $\lambda$. Using the equations

$$
\begin{array}{r}
V^{s} g_{2}=(-1)^{s} e^{i s \lambda} g_{2}+V^{s-1} \zeta-e^{i \lambda} V^{s-2} \zeta+\cdots+(-1)^{s-1} e^{i(s-1) \lambda} \zeta, \\
s=0,1,2, \cdots, \\
V^{-s} g_{2}=(-1)^{s} e^{-i s \lambda} g_{2}+e^{-i \lambda} V^{-s} \zeta-e^{-2 i \lambda} V^{-s+1} \zeta+\cdots+(-1)^{s-1} e^{-i s \lambda} V^{-1} \zeta, \\
s=0,1,2, \cdots,
\end{array}
$$

and noting that if for a given $f \varepsilon \mathfrak{B}$ with $V f=-\exp (i \lambda) f$, then $B_{\lambda-\pi / 2} f=-f$, one may write

$$
\begin{aligned}
B_{\lambda-\pi / 2} g_{2}=-g_{2} & +\sum_{r}^{\infty} \alpha_{s}\left(\lambda-\frac{\pi}{2}\right) V^{s} g_{2}+\sum_{-\infty}^{-r} \alpha_{s}\left(\lambda-\frac{\pi}{2}\right) V^{s} g_{2} \\
& -\sum_{r}^{\infty} \alpha_{s}\left(-\frac{\pi}{2}\right) g_{2}-\sum_{-\infty}^{-r} \alpha_{s}\left(-\frac{\pi}{2}\right) g_{2}+\eta
\end{aligned}
$$

where $\eta$ stands for a collection of terms of the form $\beta V^{\circ}$ \% with $|\beta| \leqq 1$. Now the norm of the first two $\sum$ terms above does not exceed $\epsilon_{3}\left\|g_{2}\right\|$. The norm of the next two $\sum$ terms does not exceed $\epsilon_{3}\left\|g_{2}\right\|$. The term $\eta$ represents a 
sum of at most $r(r+1)$ terms each of norm less than $K \epsilon_{2}\|g\| / 2$. Further, we have $B_{\lambda-\pi / 2} g_{2}=\frac{1}{2} B_{\lambda-\pi / 2} \cdot A_{\lambda-\delta} \cdot B_{\lambda+\delta}\left(f-e^{-i \lambda} V f\right)=0$. Thus if it happens that $r(r+1) K \epsilon_{2} \leqq 2 \epsilon_{3}$, then $\left\|g_{2}\right\| \leqq 2 \epsilon_{3}\left\|g_{2}\right\|+\epsilon_{3}\|g\|$, or if $\epsilon_{3}<\frac{1}{4},\left\|g_{2}\right\| \leqq 2 \epsilon_{3}\|g\|$.

Finally, in the equation

$$
\left(V-e^{i \lambda}\right)\left(g_{1}+g_{2}\right)=e^{-i \lambda} \frac{V^{2}-e^{2 i \lambda}}{2} g+\left(V-e^{i \lambda}\right) g_{2}
$$

it is seen that the norm of the first term after the equality symbol does not exceed $\epsilon_{2}\|g\| / 2$. The norm of the last term does not exceed $4 K \epsilon_{3}\|g\|$. Hence the norm of the term on the left of the equality sign does not exceed $5 K \epsilon_{3}\|g\|$.

It is a simple matter now to compute $\delta$ for the given $\epsilon$ of the theorem. Choose $\epsilon_{3}$ so that $5 K \epsilon_{3} \leqq \epsilon$. Then choose as indicated above the numbers $r, \epsilon_{2}, n, \epsilon_{1}$ and finally $\delta$ (such that $\delta \leqq \epsilon$ ), in that order. For that choice, (12) holds for all elements in $\widetilde{E}_{\lambda \delta}$.

The classes $\mathfrak{A}_{\lambda}$ will be defined. For $-\infty<\lambda<-\pi, \mathfrak{A}_{\lambda}=0$. For $\pi<\lambda<\infty$, $\mathfrak{A}_{\lambda}=0$. For $-\pi \leqq \lambda \leqq 0, \mathfrak{U}_{\lambda}$ is the intersection of the c.l.m. $\mathfrak{M}_{0}$ (see Theorem 7 ) with the c.l.m. $\mathfrak{E}_{\lambda \delta}$. For $0<\lambda \leqq \pi, \mathfrak{A}_{\lambda}$ is the intersection of the c.l.m. $\mathfrak{N}_{0}$ with the c.l.m. $\varepsilon_{\lambda \delta}$.

Proof of (a) in the theorem. Consider specifically the case $-\pi<\lambda<0$; other cases, being similar to this one, will not be discussed. Let $g=A_{\lambda-\delta} B_{\lambda+\delta} f$. Since $g \varepsilon \mathfrak{M}_{0}, A g=0$; also $A_{\lambda+\delta} g=0$. If $\lambda+\delta \geqq 0, g \varepsilon \mathfrak{E}_{\lambda+\epsilon}$, since $\mathfrak{M}_{0}=\mathfrak{F}_{0} \subset \mathfrak{E}_{\lambda+\delta} \subset \mathfrak{E}_{\lambda+\epsilon}$. If $\lambda+\delta<0$, let $f=g_{0}+g_{0}^{\prime}+g_{\lambda+\delta}+g_{\lambda+\delta}^{\prime}+h$ be the decomposition of $f$, (9), for $n=2, \lambda_{1}=0, \lambda_{2}=\lambda+\delta$. Then the decomposition of $g$ is found by operating on the decomposition of $f$ with $A_{\lambda-\delta} B_{\lambda+\delta}$. It is found that $B_{\lambda+\delta} g_{0}=0, A_{\lambda-\delta} g_{\lambda+\delta}^{\prime}=0$, $A_{\lambda-\delta} B_{\lambda+\delta} g_{0}^{\prime}=0$ (since $g \varepsilon \mathfrak{M}_{0}$ ). Thus $g \varepsilon \mathfrak{E}_{\lambda+\delta} \subset \mathbb{E}_{\lambda+\epsilon}$. Hence $\mathfrak{A}_{\lambda} \subset \mathfrak{E}_{\lambda+\epsilon}$.

To prove that $g \varepsilon \mathfrak{F}_{\lambda-\epsilon}$, it suffices to show that $g \varepsilon \mathfrak{F}_{\lambda-\delta}$. Note first that $B B_{\lambda-\delta} g=0$. Using the decomposition for $f, f=g_{0}+g_{0}^{\prime}+g_{\lambda-\delta}+g_{\lambda_{-\delta}}^{\prime}+h$, it is seen that $A_{\lambda-\delta} B_{\lambda+\delta} g_{\lambda-\delta}=0$, hence $g \varepsilon \mathfrak{F}_{\lambda-\delta}$. The proof of (b) falls out of the definition of $\mathfrak{A}_{\lambda}$.

The finite collection of manifolds $\mathfrak{A}_{\lambda_{j}}, j=1,2, \cdots, n$, which is mentioned in the theorem will now be exhibited. Let $\lambda_{1}, \cdots, \lambda_{n},-\pi<\lambda_{j}<\pi, \lambda_{j} \neq 0$, be any collection of numbers having the properties that (i) the open segments of length $2 \delta$ centered about the points $\exp \left(i \lambda_{j}\right), j=1, \cdots, n$, on the complex unit circle cover that circle; and (ii) for every $j, j=1, \cdots, n$, there exists a $k$ such that $\lambda_{j}=-\lambda_{k}$.

It will be proved that the $\mathfrak{A}_{\lambda_{j}}, j=1, \cdots, n$, together span $\mathfrak{B}$. Consider the set $M$ of numbers: $\pm 1, \pm \exp \left(i \lambda_{j}-i \delta\right), \pm \exp \left(i \lambda_{j}+i \delta\right)$; these numbers may not be distinct. Let $\mu_{1}, \mu_{2}, \cdots, \mu_{s},-\pi<\mu_{j} \leqq 0$, be distinct real numbers such that every number in the set $N$ of numbers $\pm \exp \left(i \mu_{j}\right), j=1, \cdots, s$, is found in $M$ and conversely. Let $f=\sum\left(g_{\mu_{j}}+g_{\mu_{j}}^{\prime}\right)+h$ be the decomposition (9) of $f$ which is generated by the $\mu_{j}$. As before, $h$ is in the c.l.m. spanned by the elements $k=\prod_{j=1}^{s}\left(A_{\mu_{j}}+B_{\mu_{j}}\right) l$. Note that this c.l.m. is identical with that spanned by the elements $k=\prod_{j=1}^{s}\left(A_{\mu_{j}}+B_{\mu_{j}}\right)^{t_{i} l}$ where the $t_{j}$ are any positive 
integers. In other words, $h$ is in the c.l.m. spanned by the elements

$$
k=(A+B) \prod_{j=1}^{n}\left(A_{\lambda_{j}-\delta}+B_{\lambda_{j}-\delta}\right)\left(A_{\lambda_{j}+\delta}+B_{\lambda_{j}+\delta}\right) l,
$$

$l \varepsilon \mathfrak{B}$. It will be shown first that each $g_{\mu_{j}}$ and each $g_{\mu_{j}}^{\prime}$ is in some $\mathfrak{A}_{\lambda_{j}}$; next that every term in the expansion of $k$ in (13) is in some $\mathfrak{A}_{\lambda_{j}}$.

Consider any $g_{\mu_{j}}$ or $g_{\mu_{j}}^{\prime}$; for short call it $g$, and suppose for example that $V g=\exp \left(i \lambda_{j}-i \delta\right) g$ (the case $V g= \pm g$ is tacitly assumed to have been disposed of). By condition (i) on the $\lambda_{j}$, the point $\exp \left(i \lambda_{j}+i \delta\right)$ is an interior point of some interval centered about some $\lambda_{k}$. Hence $g \varepsilon \mathfrak{E}_{\lambda_{k}} \delta$. Invoking if necessary condition (ii) on the $\lambda_{j}$, it may be seen that $g$ is in some $\mathfrak{A}_{\lambda_{l}}$.

The terms in the expansion of (13) may be written in the form

$$
T=D \prod_{j=1}^{n} D_{j} E_{j}
$$

where $D$ is either $A$ or $B, D_{j}$ is either $A_{\lambda_{j}-\delta}$ or $B_{\lambda_{j}-\delta}$, and $E_{j}$ is either $A_{\lambda_{j}+\delta}$ or $B_{\lambda_{j}+\delta}$. For some $j$, it is conceivable that $D_{j}$ and $E_{j}$ both represent an $A$; or again that both represent a $B$. Such a value of $j$ will be described as a resemblance value. Suppose that $D$ represents $A$. If all $j$ with $0<\lambda_{j}<\pi$ (note that $-\pi, 0, \pi$ are excluded as possible values for $\lambda_{j}$ ) are resemblance values, then by an argument now familiar, the transformation $T(14)$ is identically 0 in $\mathfrak{B}$, and $T l$ certainly is in all $\mathfrak{A}_{\lambda_{j}}$. Suppose that a particular $j$ is not a resemblance value. Then either the product $A_{\lambda_{j}-\delta} B_{\lambda_{j}+\delta}$ or the product $A_{\lambda_{j}+\delta} B_{\lambda_{j}-\delta}$ is present in (14). In the first case $T l \varepsilon A_{\lambda_{j}}$. If on the other hand the second case arises for all non-resemblance values $j$, then once more $T l=0$. If $D$ represents $B$, the discussion is similar. This concludes the proof of the theorem.

\section{The SPECTRUM OF $V$}

The theory so far elaborated enables one to discuss the spectrum of any w.a.p. transformation $V$. One defines as usual the point spectrum, continuous spectrum, and resolvent set for $V$ : A complex number $\alpha$ belongs to the point spectrum if $V f=\alpha f$ for some $f \neq 0$. The number $\alpha$ belongs to the continuous spectrum if the transformation $V-\alpha I$ possesses an unbounded inverse whose domain is dense in $\mathfrak{B}$. Finally the value $\alpha$ belongs to the resolvent set if the transformation $V-\alpha I$ possesses a bounded inverse whose domain is $\mathfrak{B}$ in its entirety. The above classes are mutually exclusive but are not for a general (non-w.a.p.) transformation all inclusive. It will be shown below that they cover all the possibilities for $V$.

Relative to the manifold pairs $\left\{\mathfrak{E}_{\lambda}, \mathfrak{F}_{\lambda}\right\}$, one may define certain classes of numbers in the following fashion: The numbers $\lambda$ for which there exists a $\delta>0$ such that $\mathbb{F}_{\lambda-\delta}=\mathfrak{F}_{\lambda+\delta}, \mathfrak{F}_{\lambda-\delta}=\mathfrak{F}_{\lambda+\delta}$ constitute the points of constancy of $\left\{\mathfrak{E}_{\lambda}, \mathfrak{F}_{\lambda}\right\}$. For instance the numbers $\lambda<-\pi$ and $\lambda>\pi$ are points of constancy. The num- 
bers $\lambda$ which are not points of constancy but for which $\mathfrak{F}_{\lambda-0}=\mathfrak{F}_{\lambda}$ will constitute the points of continuity of $\left\{\mathfrak{E}_{\lambda}, \mathfrak{F}_{\lambda}\right\}\left({ }^{12}\right)$. The numbers $\lambda$ for which $\mathfrak{F}_{\lambda-0} \neq \mathfrak{F}_{\lambda}$ will constitute the points of discontinuity of $\left\{\mathcal{E}_{\lambda}, \mathfrak{F}_{\lambda}\right\}$. Thus each real $\lambda$ belongs to one and only one of these three types. The striking relationship of the character of $\left\{\mathfrak{F}_{\lambda}, \mathfrak{F}_{\lambda}\right\}$ to the spectrum of $V$ is exposed in the following theorem.

THEOREM 9. Let $V$ be a weakly almost-periodic transformation, and let $\left\{\mathfrak{E}_{\lambda}, \mathfrak{F}_{\lambda}\right\}$ be the manifold pairs associated with it in Theorem 7. Let $\alpha$ be any complex number. Then if $|\alpha| \neq 1, \alpha$ belongs to the resolvent set of $V$. If the real number $\lambda$ is restricted to the interval $-\pi<\lambda<\pi$, then

(a) The value $\alpha=\exp (i \lambda)$ belongs to the point spectrum if and only if $\lambda$ is a point of discontinuity of $\left\{\mathbb{E}_{\lambda}, \mathfrak{F}_{\lambda}\right\}$.

(b) The value $\alpha=\exp (i \lambda)$ belongs to the continuous spectrum if and only if $\lambda$ is a point of continuity of $\left\{\mathfrak{E}_{\lambda}, \mathfrak{F}_{\lambda}\right\}$.

(c) The value $\alpha=\exp (i \lambda)$ belongs to the resolvent set if and only if $\lambda$ is a point of constancy of $\left\{\mathfrak{E}_{\lambda}, \mathfrak{F}_{\lambda}\right\}$.

The value $\alpha=\exp (-i \pi)=\exp (i \pi)$ belongs to the resolvent set if and only if $-\pi$ and $\pi$ are points of constancy of $\left\{\mathfrak{E}_{\lambda}, \mathfrak{F}_{\lambda}\right\}$. The value $\exp (i \pi)$ belongs to the point spectrum if and only if $\pi$ is a point of discontinuity of $\left\{\mathfrak{E}_{\lambda}, \mathfrak{F}_{\lambda}\right\}$. In all other cases, $\exp (i \pi)$ belongs to the continuous spectrum.

Suppose first that $|\alpha|>1$. Then $\left|\left(\alpha^{-1} V\right)^{n}\right| \leqq|\alpha|^{-n} K$ where as before $\left|V^{n}\right| \leqq K$. Thus by Theorem $4^{\prime}$, the transformation $\alpha^{-1} V-I$ possesses a bounded inverse; hence $V-\alpha I$ does likewise.

Now let $|\alpha|<1$. By the reasoning just given $V^{-1}(V-\alpha I)=I-\alpha V^{-1}$ possesses a bounded inverse. Hence $V-\alpha I$ does likewise.

If $|\alpha|=1$, then by Theorem 4 . if $\left(\alpha^{-1} V-I\right) f=0$ has no non-trivial solutions, the elements $\left(\alpha^{-1} V-I\right) f, f \varepsilon \mathfrak{B}$, are dense in $\mathfrak{B}$. Thus if $\alpha$ does not belong to the point spectrum of $V$, it belongs either to the continuous spectrum or to the resolvent set. For if the range of $S=\alpha^{-1} V-I$ is $\mathfrak{B}$ in its entirety, $S$ gives a one-to-one mapping of $\mathfrak{B}$ into itself which is continuous in one direction; therefore the mapping is continuous in the reverse direction. In this case $\alpha$ belongs to the resolvent set. If the range of $S$ is dense in $\mathscr{B}$ but is not $\mathscr{B}$, then $S^{-1}$ cannot be bounded; for if $S^{-1}$ were bounded, the range of $S$ could be extended to the entire space $\mathfrak{B}$. In this case $\alpha$ is in the continuous spectrum.

Toward (a). Suppose $\alpha=\exp (i \lambda)$ belongs to the point spectrum and that $V f=\exp (i \lambda) f, f \neq 0$. Then it is easy to conclude that $f \varepsilon \mathfrak{F}_{\mu}, \mu<\lambda$. On the other hand $f \varepsilon \mathbb{E}_{\lambda}$, hence $f$ is not in $\mathfrak{F}_{\lambda}$. Thus $\mathfrak{F}_{\lambda-0} \neq \mathfrak{F}_{\lambda}$.

Let $f \varepsilon \mathfrak{F}_{\lambda-0}, f \notin \mathfrak{F}_{\lambda}$; and let $f=g_{0}+g_{0}^{\prime}+g_{\lambda}+g_{\lambda}^{\prime}+h$ be a partition (9) of $f$. Assume for the sake of definiteness that $-\pi<\lambda<0$. Since $B B_{\mu} f=0,-\pi<\mu<\lambda$, $B B_{\lambda} f=0$ by virtue of the continuity of $B B_{\mu}$. Since $f \notin F_{\lambda}, g_{\lambda} \neq 0$. Thus there exists an element $g_{\lambda} \neq 0$ such that $V g_{\lambda}=\alpha g_{\lambda}$ and $\alpha$ is in the point spectrum of $V$.

(12) $\mathfrak{F}_{\lambda-0}$ shall be defined to be the intersection of all $\mathfrak{F}_{\mu}$ with $\mu<\lambda: \mathfrak{F}_{\lambda-0}=\prod_{\mu<\lambda} \mathfrak{F}_{\mu}$. 
Toward (b). Let $\exp (i \lambda)$ be in the continuous spectrum. It will be shown that for any $\delta>0$ with $-\pi<\lambda-\delta<\lambda+\delta<\pi$ there exists an element $g=A_{\lambda-\delta} B_{\lambda+\delta} f \neq 0$. Since $g \varepsilon \mathcal{E}_{\lambda+\delta}$ and $g \varepsilon \mathfrak{F}_{\lambda-\delta}$, the equation $\mathcal{E}_{\lambda+\delta}=\mathbb{E}_{\lambda-\delta}$ is impossible; thus $\lambda$ is not a point of constancy of $\left\{\mathbb{E}_{\lambda}, \mathfrak{F}_{\lambda}\right\}$. Since $\lambda$ is not a point of discontinuity by (a), $\lambda$ must be a point of continuity of $\left\{\mathbb{E}_{\lambda}, \mathfrak{F}_{\lambda}\right\}$.

Since $\exp (i \lambda)$ is in the continuous spectrum, it is possible, given an integer $N$ and an $\epsilon>0$, to find an $f \neq 0$ such that $\left\|\left(V^{n}-\exp (i n \lambda)\right) f\right\| \leqq \epsilon\|f\|$, $n= \pm 1, \pm 2, \cdots, \pm N$. Thus for such an $f$ the elements

$$
\left(A_{\lambda-\delta}-\sin \delta\right) f, \quad A_{\lambda+\delta} f, \quad\left(B_{\lambda+\delta}+\sin \delta\right) f, \quad B_{\lambda-\delta} f,
$$

have a norm small at will compared to that of $f$. This is proved as in Theorem 8 in the discussion preceding (a). Thus an $f \varepsilon \mathfrak{B}$ can be found such that $A_{\lambda-\delta} B_{\lambda+\delta} f+\sin ^{2} \delta f$ has a norm small at will compared to that of $f$. This means that $A_{\lambda-\delta} B_{\lambda+\delta} f \neq 0$.

In proving the converse, one starts with the fact that the simultaneous equations $\mathfrak{E}_{\lambda+\delta}=\mathfrak{E}_{\lambda-\delta}, \mathfrak{F}_{\lambda+\delta}=\mathfrak{F}_{\lambda-\delta}$ are impossible for any $\delta>0$. Assume that the former equation does not hold, hence that there exists an $f \neq 0$ such that $f \varepsilon \varepsilon_{\lambda+\delta}$, $f \notin \mathbb{E}_{\lambda-\delta}$. Assume for immediate convenience that $-\pi<\lambda-2 \delta<\lambda+2 \delta<0$. If $g=A_{\lambda-2 \delta} f=0, f \varepsilon \mathcal{E}_{\lambda-\delta}$ which is impossible. If $B_{\lambda+2 \delta} g=0, g \varepsilon \mathfrak{F}_{\lambda+\delta}$ which is impossible since, like $f, g \varepsilon \mathbb{E}_{\lambda+\delta}$. Since $\delta$ is arbitrary, by Theorem 8, (12), $V$ - $\exp (i \lambda) I$ does not possess a bounded inverse, hence exp $(i \lambda)$ is either in the continuous spectrum or in the point spectrum. The latter possibility is barred by the proof of (a) of the present theorem. This demonstration has been carried through for a sample case; other cases may be handled in a similar manner.

Toward (c). Proof here is obtained by applying the exclusion principle. The statements in the theorem concerning the value $\alpha=\exp (-i \pi)=\exp (i \pi)$ will not be discussed as the methods of proof here needed are not very different from those already indicated.

\section{The TRANSFormation $H$}

Throughout the present chapter, $V$ will be a w.a.p. transformation for which the transformation $(V+I)^{-1}$ exists, that is, for which $\alpha=-1$ is not in the point spectrum. A very important function, $H$, of $V$ is defined by

$$
H=-i(V-I)(V+I)^{-1} \text {. }
$$

The meaning of (15) is to be precisely this: $H$ is defined for all elements $f$ of the form $f=(V+I) g, g$ arbitrary in $\mathfrak{B}$; and $H f=-i(V-I) g$. The transformation $H$ enjoys closure. Let the sequence $\left\{f_{n}\right\}$ be in the domain of $H$. Let $f_{n} \rightarrow f$, and $H f_{n} \rightarrow f^{\prime}$. Then $f$ is in the domain of $H$ and $H f=f^{\prime}$. For writing $f_{n}=(V+I) g_{n}$ and $H f_{n}=-i(V-I) g_{n}$, it is clear that the convergence of $\left\{f_{n}\right\}$ and of $\left\{H f_{n}\right\}$ implies that of $\left\{g_{n}\right\}$. Let $g_{n} \rightarrow g$, then $f=(V+I) g$ and $f^{\prime}=\lim \left[-i(V-I) g_{n}\right]=-i(V-I) g=H f$. If $(V+I)^{-1}$ is bounded, then $H$ is 
bounded. Conversely, if $H$ is bounded, since it is closed and defined over a dense set in $\mathfrak{B}$, its domain of definition is $\mathfrak{B}$ in its entirety; thus $(V+I)^{-1}$ is bounded. Thus $H$ is bounded if and only if there exists a $\lambda<\pi$ such that $\mathfrak{E}_{\lambda}=\mathfrak{B}, \mathfrak{F}_{\lambda}=0, \mathfrak{E}_{-\lambda}=0, \mathfrak{F}_{-\lambda}=\mathfrak{B}$.

Let $\lambda$ and $\mu$ be real variables related by $\lambda=\tan \mu / 2,-\pi<\mu<\pi$. Consider the manifold pairs $\left\{\mathfrak{F}_{\lambda}, \mathfrak{F}_{\lambda}\right\}$ of Theorem 7 with $\lambda$ restricted to $-\pi<\lambda<\pi$. Construct the pairs of manifolds $\left\{\mathscr{E}_{\lambda}^{\prime}, \mathfrak{F}_{\lambda}^{\prime}\right\},-\infty<\lambda<\infty$, where $\mathbb{E}_{\lambda}^{\prime}=\mathbb{E}_{\mu}$, $\mathfrak{F}_{\lambda}{ }^{\prime}=\mathfrak{F}_{\mu}$. Then all the statements of Theorem 7 except $(\mathrm{d})$ are valid if $\left\{\mathbb{E}_{\lambda}, \mathfrak{F}_{\lambda}\right\}$ is replaced by $\left\{\mathscr{E}_{\lambda}^{\prime}, \mathfrak{F}_{\lambda}^{\prime}\right\}$. In place of $(\mathrm{d})$, one may write $\prod_{\lambda} \mathfrak{F}_{\lambda}^{\prime}=0, \prod_{\lambda} \mathfrak{F}_{\lambda}^{\prime}=0$, where the product is formed over all real $\lambda$. The first formula is established with the help of the fact that $\mathfrak{F}_{\pi-0}=\mathfrak{F}_{\pi}=0$. The second may be verified readily.

The point spectrum, continuous spectrum, and resolvent set of $H$ are defined as for $V$ (Chapter $\mathrm{V})$. Reducibility of $H$ by a manifold pair $\{\mathfrak{M}, \mathfrak{N}\}$ could be defined as for $V$, but the appearance of unboundedness creates difficulties which lead to the abandonment of this idea. Instead, the pair $\{\mathfrak{M}, \mathfrak{N}\}$ will be said to reduce $H$ if it reduces $V$. If $\mathfrak{M}$ and $\mathfrak{N}$ are disjoint, this implies that $H f$ is in $\mathfrak{M}$ (or $\mathfrak{N}$ ) whenever $f$ is in $\mathfrak{M}$ (or $\mathfrak{N}$ ). For let $f \varepsilon \mathfrak{M}, f=(V+I) g$, $g=g_{1}+g_{2}$ with $g_{1} \varepsilon \mathfrak{M}, g_{2} \varepsilon \mathfrak{R}$. Then since $\{\mathfrak{M}, \mathfrak{R}\}$ reduces $V,(V+I) g_{2} \varepsilon \mathfrak{R}$; but $(V+I) g_{2}=f-(V+I) g_{1} \varepsilon \mathfrak{M}$. Thus $(V+I) g_{2}=0, g_{2}=0$. Hence Hf $\varepsilon \mathfrak{M}$.

The relationship of $\left\{\mathcal{E}_{\lambda}^{\prime}, \mathfrak{F}_{\lambda}^{\prime}\right\}$ to $H$ is brought to light in the next two theorems. The first establishes for $H$ an integral representation (1).

THEOREM 10. Let $H$ be the transformation $H=-i(V-I)(V+I)^{-1}$ and let $\left\{\mathcal{E}_{\lambda}^{\prime}, \mathfrak{F}_{\lambda}^{\prime}\right\},-\infty<\lambda<\infty$, be the manifold pairs constructed above. Let a real number $\epsilon>0$ be given. Then for every real $\lambda$ there exists a closed linear manifold $\mathfrak{D}_{\lambda}$ such that:

(a) The manifold $\mathfrak{D}_{\lambda}$ lies in $\mathfrak{E}_{\lambda_{+\epsilon}^{\prime}}^{\prime}$ and $\mathfrak{F}_{\lambda_{-\epsilon}}^{\prime}$.

(b) If $f \boldsymbol{\varepsilon} \mathfrak{D}_{\lambda}$, then $H f$ exists and

$$
\|(H-\lambda I) f\| \leqq \epsilon\|f\| .
$$

Furthermore, there exists a denumerable collection of values $\lambda$, viz., $\lambda_{1}, \lambda_{2}, \cdots$, such that the space $\mathfrak{B}$ is spanned by the manifolds $\mathfrak{D}_{\lambda_{j}}, j=1,2, \cdots$. If $H$ is bounded, this infinite collection of $\lambda_{j}$ may be replaced by a finite set.

The definition of the c.l.m. $\mathfrak{D}_{\lambda}$ must be prefaced by a brief discussion. Consider first the c.l.m. $\mathfrak{A}_{\mu}$ of Theorem 8 for a $\mu$ such that $-\pi<\mu<\pi$. Specifically, let $\mathfrak{A}_{\mu}$ satisfy the conditions of Theorem 8 relative to an $\epsilon_{1}<2 \cos \mu / 2$; further let the $\delta$ of $\mathfrak{A}_{\mu}$ satisfy $-\pi<\mu-\delta<\mu+\delta<\pi$. Then the transformation $V+I$ transforms $\mathfrak{A}_{\mu}$ into itself in a one-to-one bicontinuous manner. This will be proved by showing first that the range of $V+I$ (defined over $\mathfrak{A}_{\mu}$ ) is a c.l.m.; subsequently that this range is dense in $\mathfrak{A}_{\mu}$.

By the definition of $\mathfrak{A}_{\mu},(V+I) g \varepsilon \mathfrak{A}_{\mu}$ if $g \varepsilon \mathfrak{A}_{\mu}$. Let $\left\{g_{n}\right\}$ be a sequence in $\mathfrak{U}_{\mu}$, let $f_{n}=(V+I) g_{n}$, and suppose that $f_{n} \rightarrow f$. Writing $V g_{n}=\exp (i \mu) g_{n}+\eta_{n}$, it follows from (12) that $\left\|\eta_{n}\right\| \leqq \epsilon_{1}\left\|g_{n}\right\|,\left\|\eta_{n}-\eta_{m}\right\| \leqq \epsilon_{1}\left\|g_{n}-g_{m}\right\|$. Thus $\left\|g_{n}-g_{m}\right\|$ 
$\leqq\left(2 \cos \mu / 2-\epsilon_{1}\right)^{-1}\left\|f_{n}-f_{m}\right\|,\left\{g_{n}\right\}$ is convergent, say $g_{n} \rightarrow g$ and $(V+I) g=f$. Hence the range of $V+I$ is a c.l.m.

Now let $g$ be arbitrary in $\mathfrak{A}_{\mu}$. Using the identity $(V+I) g=(\exp (i \mu)+1) g$ $+(V-\exp (i \mu)) g$, and writing $\alpha=(\exp (i \mu)+1)^{-1}$, it is seen that

$$
\begin{aligned}
(V+I)\left[\alpha I-\alpha^{2}\left(V-e^{i \mu}\right)+\alpha^{3}\left(V-e^{i \mu}\right)^{2}-\cdots\right. & \left.+(-1)^{n+1} \alpha^{n}\left(V-e^{i \mu}\right)^{n-1}\right] g \\
& =g+(-1)^{n+1} \alpha^{n}\left(V-e^{i \mu}\right)^{n} g .
\end{aligned}
$$

The norm of this last element does not exceed $\epsilon_{1}^{n}(2 \cos \mu / 2)^{-n}\|g\|$, hence is small at will if $n$ is sufficiently large. This proves that the range of $V+I$ is $\mathfrak{A}_{\mu}$.

The transformation $H$ is defined and bounded on $\mathfrak{A}_{\mu}$. To compute the norm on $\mathfrak{A}_{\mu}$ of $H-\lambda I$ where $\lambda=\tan \mu / 2$, write $H f=-i(V-I) g$ with $(V+I) g=f$. Calculation shows that $(H-\lambda I) f=-(i+\lambda)[V-\exp (i \mu)] g$. Since $\| g_{i}$ $\leqq\left(2 \cos \mu / 2-\epsilon_{1}\right)^{-1}|| f||,|H-\lambda I| \leqq \epsilon$ if $\epsilon_{1}(1+|\lambda|)\left(2 \cos \mu / 2-\epsilon_{1}\right)^{-1} \leqq \epsilon$, where $\epsilon$ is the constant mentioned in the statement of the present theorem.

The definition of $\mathfrak{D}_{\lambda}$ is: $\mathfrak{D}_{\lambda}=\mathfrak{A}_{\mu}, \lambda=\tan \mu / 2$. Note that (b) has been established. It was demonstrated in Theorem 8 that $\mathfrak{A}_{\mu}$ lies in $\mathfrak{E}_{\mu+\delta}$ as well as in $\mathfrak{F}_{\mu-\delta}$. Thus $\mathfrak{D}_{\lambda}$ lies in $\mathfrak{E}_{\lambda_{1}}^{\prime}, \mathfrak{F}_{\lambda_{2}}^{\prime}$, where $\lambda_{1}=\tan (\mu+\delta) / 2, \lambda_{2}=\tan (\mu-\delta) / 2$. By choosing $\delta$ properly, one may make $\lambda_{1} \leqq \lambda+\epsilon, \lambda_{2} \geqq \lambda-\epsilon$. This proves (a).

The collection of values $\lambda_{1}, \lambda_{2}, \cdots$ will be exhibited. The procedure just outlined for the choice of $\mathfrak{D}_{\lambda}=\mathfrak{A}_{\mu}$ embeds every point exp $(i \mu)$ on the complex unit circle (except the point $\mu=\pi$ ) in an interval of varying length $2 \delta, \delta$ depending on $\mu$. Let $\mu_{1}, \mu_{2}, \cdots,-\pi<\mu_{j}<\pi, \mu_{j} \neq 0$, be any sequence of numbers having the properties: (i) The open intervals of length $2 \delta$ centered about $\exp (i \mu)$ cover completely the unit circle from which the point $\mu=\pi$ has been excluded; (ii) for every $\mu_{j}$ there exists a $\mu_{k}$ such that $\mu_{j}=-\mu_{k}$. The sequence $\lambda_{1}, \lambda_{2}, \cdots$ is defined by $\lambda_{j}=\tan \mu_{j} / 2, j=1,2, \cdots$.

Let $F$ be any linear functional in (B) such that $F \perp \mathfrak{D}_{\lambda_{j}}, j=1,2, \ldots$. It will be proved that $F=0$, hence that the c.l.m. spanned by the $\mathfrak{D}_{\lambda_{j}}$ is $\mathfrak{B}$. Let $\nu$ be any number, $-\pi<\nu<0$. Let $n$ be an integer such that the semicircle $\exp (i x), \nu \leqq x \leqq \nu+\pi$ is covered by the above described intervals centered about the points $\exp \left(i \mu_{j}\right), j=1,2, \cdots, n$. Then the manifolds $\mathfrak{M}_{\nu}, \mathfrak{A}_{\mu_{j}}$, $j=1, \cdots, n, \operatorname{span} \mathfrak{B}\left({ }^{13}\right)$. Now for $\bar{A}_{\nu} F\left(\bar{A}_{\nu}\right.$ is the transformation adjoint to $\left.A_{\nu}\right)$, $\bar{A}_{\nu} F \perp \mathfrak{M}_{\nu}$ as well as $\bar{A}_{\nu} F \perp \mathfrak{A}_{\mu_{j}}, j=1, \cdots, n$. Hence $\bar{A}_{\nu} F=0$. Noting that $\nu$ is arbitrary, and letting $\nu \rightarrow 0$, one has $\bar{A} F=0$. A very similar argument using a $\nu$ with $0<\nu<\pi$ shows that $\bar{B} F=0$. Thus for $F, \bar{V} F=-F$. By Theorem 3 , the collection of elements $F$ having the latter property is the orthogonal complement of the c.l.m. $\mathfrak{B}$ spanned by the elements $(V+I) f$. Therefore $F=0$.

If $H$ is bounded, the points $\mu=-\pi$ and $\mu=\pi$ are points of constancy of $\left\{\mathfrak{E}_{\mu}, \mathfrak{F}_{\mu}\right\}$ and it is easy to see that only a finite number of the manifolds $\mathfrak{A}_{\mu_{j}}$ contribute effectively to the spanning of $\mathfrak{B}$.

${ }^{(13)}$ The argument needed to establish this is similar in spirit to that given in the last paragraphs of Chapter IV. To the collection of transformations $A_{\mu_{j} \pm \delta}, B_{\mu_{j} \pm \delta}, A$, and $B$, one must adjoin $A_{\nu}, B_{\nu}$. 
In the next theorem, the spectral characteristics of $H$ are completely described by the structure of $\left\{\mathcal{E}_{\lambda}^{\prime}, \mathfrak{F}_{\lambda}^{\prime}\right\}$.

Theorem 11. Let $H$ and $\left\{\mathbb{E}_{\lambda}^{\prime}, \mathfrak{F}_{\lambda}^{\prime}\right\}$ be as in Theorem 10. Let $\alpha=\lambda+\lambda^{\prime} i$ be any complex number. The value $\alpha$ is in the resolvent set of $H$ whenever $\lambda^{\prime} \neq 0$. The transformation $V$ which generates $H$ is completely determined by $H$ :

$$
V=-(H-i I)(H+i I)^{-1} \text {. }
$$

If $\lambda^{\prime}=0$, then

(a) The value $\alpha=\lambda$ belongs to the point spectrum of $H$ if and only if $\lambda$ is a point of discontinuity of $\left\{\mathbb{E}_{\lambda}^{\prime}, \mathfrak{F}_{\lambda}^{\prime}\right\}$.

(b) The value $\alpha=\lambda$ belongs to the continuous spectrum of $H$ if and only if $\lambda$ is a point of continuity of $\left\{\mathfrak{F}_{\lambda}^{\prime}, \mathfrak{F}_{\lambda}^{\prime}\right\}$.

(c) The value $\alpha=\lambda$ belongs to the resolvent set of $H$ if and only if $\lambda$ is a point of constancy of $\left\{\mathbb{E}_{\lambda}^{\prime}, \mathfrak{F}_{\lambda}^{\prime}\right\}$.

Write $f=(V+I) g, \quad H_{\alpha} f=(H-\alpha I) f=-(i+\alpha) V g+(i-\alpha) g$. If $\alpha=-i$, $H_{-i} f=0$ implies $f=0$. The range of $H_{-i}$ is $\mathfrak{B}$; if $\left\{H_{-i} f_{n}\right\}$ is convergent, then $\left\{f_{n}\right\}$ is convergent. Thus $\alpha=-i$ is in the resolvent set of $H$. Henceforth let $\alpha \neq-i$. Then $H_{\alpha} f=-(i+\alpha)(V-\beta I) g$, where $\beta=(i-\alpha)(i+\alpha)^{-1}$. If $\lambda^{\prime} \neq 0$, then $|\beta| \neq 1$. In this case $H_{\alpha} f=0$ implies $g=f=0$ by Theorem 9 . The range of $H_{\alpha}$ is again $\mathscr{B}$ since $\beta$ is in the resolvent set of $V$. For the same reason, the convergence of $\left\{H_{\alpha} f_{n}\right\}$ with $f_{n}=(V+I) g_{n}$ implies that of $\left\{g_{n}\right\}$ and hence that of $\left\{f_{n}\right\}$; thus $\alpha$ is in the resolvent set of $H$.

Úsing the above notation, it is seen that $(H+i I)^{-1} g=(2 i)^{-1} f ;(H-i I) f=$ $-2 i \mathrm{Vg}$. This establishes (17).

Now let $\lambda^{\prime}=0, \alpha=\lambda$; then $|\beta|=1$, or more precisely $\beta=\exp (i \mu)$ with $\tan \mu / 2=\lambda$. Suppose $H_{\lambda} f=0$; then $V g=\beta g$-and conversely. Thus $\lambda$ is in the point spectrum of $H$ if and only if $\beta$ is in the point spectrum of $V$. Note that the range of $H_{\lambda}$ is dense in $\mathfrak{B}$ if and only if $\lambda$ is not in the point spectrum of $H$. Suppose the range of $H_{\lambda}$ is dense in $\mathfrak{B}$ but is not $\mathfrak{B}$. Since $H_{\lambda}$ is a closed transformation, $\left(H_{\lambda}\right)^{-1}$ cannot be bounded; thus $\lambda$ is in the continuous spectrum of $H$. In this case, $\beta$ is in the continuous spectrum of $V$. Conversely, if $\beta$ is in the continuous spectrum of $V, \lambda$ is in that of $H$. Finally, if the range of $H_{\lambda}$ is $\mathscr{B}$, then $\beta$ is in the resolvent set of $V$ and the convergence of $\left\{H_{\lambda} f_{n}\right\}$ implies that of $\left\{g_{n}\right\}$ and $\left\{f_{n}\right\}$. Thus if the range of $H_{\lambda}$ is $\mathfrak{B}, \lambda$ is in the resolvent set of $H$. Hence $\lambda$ is in the resolvent set of $H$ if and only if $\beta$ is in the resolvent set of $V$.

It appears that the spectral character of $H_{\lambda}$ is identical with that of $V_{\mu}-I$. Since the relation between $\lambda$ and $\mu$ is $\lambda=\tan \mu / 2$, and since $\left\{\mathscr{E}_{\lambda}^{\prime}, \mathfrak{F}_{\lambda}^{\prime}\right\}$ and $\left\{\mathfrak{F}_{\mu}, \mathfrak{F}_{\mu}\right\}$ are related in the same way, the verification of statements (a), (b), and (c) is shouldered by Theorem 9 .

Barnard College, Columbia University, New York, N. Y. 